\title{
DEFORMATION AND TEXTURE EVOLUTION OF OFHC COPPER DURING DYNAMIC TENSILE EXTRUSION
}

\author{
M. Hörnqvist ${ }^{1}$, N. Mortazavi ${ }^{1}$, M. Halvarsson ${ }^{1}$, A. Ruggiero $^{2}$, G. Iannitti ${ }^{2}$, N. Bonora $^{2}$ \\ ${ }^{1}$ Applied Physics, Chalmers University of Technology, Gothenburg, Sweden \\ ${ }^{2}$ Civil and Mechanical Engineering Department, University of Cassino and Southern Lazio, \\ Cassino, Italy.
}

\begin{abstract}
During dynamic tensile extrusion (DTE) the material is subjected to a complex deformation history, including high strain rates, large strains and elevated temperatures. This technique provides unique means to explore material performance under extreme conditions. In this work, the microstructural evolution of $99.98 \%$ commercially pure copper during DTE test was investigated by means of electron backscatter diffraction (EBSD). The investigation was focused on the segment of the extruded jet that remained in the die, since numerical simulation showed that material points along the longitudinal axis of such segment correspond to different stages of a common temperature compensated deformation history. Therefore, post mortem microstructure information extracted at different locations along the centre line is equivalent to in situ real-time measurement during the deformation process. EBSD investigations along the centre line showed a progressive elongation of the grains, and an accompanying development of a strong $<001>+<111>$ dual fibre texture. Meta-dynamic discontinuous dynamic recrystallization (DRX) occurred at larger strains, and it was demonstrated that nucleation occurred during straining, while subsequent grain growth took place during post-deformation cooling in the die. According to strain energy minimization arguments, the recrystallization resulted in an increased $<001>$ texture component. The critical strain for recrystallization was well predicted from a power-law dependence on the Zener-Hollomon parameter, including grain size dependence and a temperature dependent activation energy. In addition, it was shown that $<001>$ and $<111>$ oriented grains develop different dislocation substructures during straining, exhibiting elongated cells/micro-bands and typical cell structures, respectively. The present results also confirm that dynamic tensile ductility increases with decreasing initial grain size as a result of grain refinement and lowering of dislocation and twin densities during DRX.
\end{abstract}


Keywords: Dynamic tensile extrusion, copper, DRX, EBSD, texture

\section{INTRODUCTION}

In many engineering applications and technological processes, materials are subjected to severe operating conditions, such as large plastic deformation, elevated temperature, high strain rates and high pressure. In hot metal working - such as forging, rolling, extrusion, wire drawing and sheet metal forming - metals and alloys undergo significant plastic deformation (strain of 0.1-1.0) at temperatures higher than the recrystallization temperature, at strain rates ranging from 1.0 up to $100 \mathrm{~s}^{-1}$ and static pressure varying from a few MPa up to several hundreds of MPa. In defense and aerospace applications, materials that undergo high-velocity impact can be exposed to even more extreme conditions. In shaped charge jet formation the plastic strain can reach $1000 \%$, the strain rate is up to $10^{6} \mathrm{~s}^{-1}$, the temperature generated by the shock compression is in excess of $0.7 \mathrm{~T}_{\mathrm{m}}$ and the pressure approaching $100 \mathrm{GPa}$ [1].

Knowing the material response under such conditions is fundamental for advanced modelling development to support better and more accurate design. Current laboratory testing techniques - such as dynamic traction/compression with Hopkinson pressure bar and gas gun experiments - allow investigation of material behavior only under limited combinations of strain, pressure, strain rate and temperature [2]. In the last decade, the use of numerical simulation to probe material properties and validate constitutive modelling has been proposed [3] and successfully used in a number of cases [4-6]. This approach consists in performing "validation" tests - in which the governing variables (strain, strain rate, pressure, temperature, etc.) are in the range of interest although they vary during the test following complex paths - and comparing the predicted and the post mortem measured response obtained for several "validation" metrics. Such type of validation tests includes Taylor anvil impact [7] , symmetrical Taylor or rod-on-rod impact test [8], scaled energy impact tests, expanding ring test, etc.

Recently, a new test, called dynamic tensile extrusion (DTE), was proposed by Gray III et al. [9]. In this test a projectile is launched into a conical die with an exit hole smaller than the projectile diameter. During impact with the die, the projectile is subjected to both pressure and shear stress waves, and the material is adiabatically deformed and dynamically extruded at higher velocity than the initial flight speed. Tests performed on metals showed that strains in excess of $500 \%$, strain rates of the order of $10^{6} \mathrm{~s}^{-1}$, temperatures up to $1200 \mathrm{~K}$ and pressures approaching $5 \mathrm{GPa}$ can be achieved. This test has been used to investigate the dynamic response 
of both metals (oxygen-free high conductivity (OFHC) copper [10], tantalum [11] and zirconium [12]) and polymers [13].

For OFHC copper, Gray III et al. [9] investigated the role of the initial grain size in the dynamic extrusion finding that the ductility, measured as the overall length of the extruded fragments, increases with decreasing initial grain size. Recently, Park et al. [14] investigated the response to DTE in coarse and ultrafine grained OFHC copper. In contrast, they found that the overall ductility was larger in the coarse grained than in the ultrafine grained copper. In addition, they found no evidence of recrystallization in coarse grained copper while it was indeed observed in the ultrafine grained material.

Several authors have attempted to simulate OFHC copper deformation during DTE testing. Gray III et al. [9] simulated DTE test using MESA 2D explicit Eulerian code. In that case, the mechanical threshold stress (MTS) model was used to simulate copper at high strain rate and large strain. However, their results were only indicative of the fragmentation occurring during the dynamic extrusion. Simulation with CTH multi-material Eulerian code and EPIC Lagrangian finite element code were performed at Eglin AFB [15]. Again, these results were similar to those obtained with MESA indicating an inherent difficulty of the Eulerian formulation in following jet formation and fragmentation. Carlucci et al. [16] investigated the possibility to reproduce DTE test with ABAQUS explicit Lagrangian code. In this study, the Johnson and Cook model for copper was used and the die was simulated as a rigid body. They results were in a general good agreement with the experimental results obtained in [9] although a quantitative comparison was not provided. Iannitti et al. [17], using direct integration algorithm in MSC MARC implicit finite element code, simulated the DTE test to validate material constitutive modelling. In particular, they used Johnson and Cook model, modified for high strain rates, coupled with a microstructure model to account for grain size evolution. They showed the possibility to accurately predict the shape and numbers of ejected fragments as a function of the initial grain size, in accordance with Gray III et al. [9] results, emphasizing the necessity to account for microstructure evolution under such extreme loading conditions. Furthermore, these computational results indicated that, in the DTE test, microstructure evolution could also be used as an additional validation metric for models that incorporate microstructural variables.

The purpose of this study was to investigate microstructure evolution of OFHC copper during DTE to provide measures that could be used to support constitutive model verification and validation. In particular, the post extrusion texture evolution in the fragment remaining in the die, as well as the development of the deformed microstructure, was quantified to 
understand the deformation path of OFHC copper during dynamic tensile extrusion. To correlate Electron Backscatter Diffraction (EBSD) measurements with continuum state variable evolution, a finite element model (FEM) was calibrated reproducing the number and shape of extruded fragments.

\section{EXPERIMENTAL}

\subsection{Material and analytical techniques}

Oxygen-free high conductivity (OFHC) copper was obtained in the form of half-hardened (H02) bars. The material commercial purity was $99.98 \%$, although quantitative chemical analysis revealed a purity of at least 99.99\%. Projectiles for DTE testing, in the form hemispherical head bullets, were machined and annealed for 30 minutes at $400^{\circ} \mathrm{C}$ in an inert Argon atmosphere.

EBSD examinations were performed for microstructural and micro-textural analyses of the DTE sample using a LEO Ultra 55 Field Emission Gun Scanning Electron Microscope (FEG-SEM) equipped with an HKL Channel 5 EBSD system with a Nordlys II detector. Samples were electrochemically polished in a solution of $170 \mathrm{~g}$ chromic acid $\left(\mathrm{CrO}_{3}\right)$ and $830 \mathrm{~mL}$ water following the standard metallographic preparation techniques prior to the EBSD measurements. The instrument was operated at a voltage of $20 \mathrm{kV}$ with $120 \mu \mathrm{m}$ aperture size and a working distance of $13 \mathrm{~mm}$. An area of approximately $300 \times 300 \mu \mathrm{m}^{2}$ was scanned with a step size of 0.6 $\mu \mathrm{m}$. For identification of twin boundaries ( $\Sigma 3$, ideal misorientation of $60^{\circ}$ ) a misorientation range of $58-62^{\circ}$ was used.

Transmission Electron Microscopy (TEM) was employed to shed light on the dislocation structures in the deformed grains using an FEI TITAN 80-300 TEMSTEM instrument equipped with a FEG. The TEM thin foils were prepared by the site-specific in-situ lift-out procedure using an FEI Versa dual-beam workstation, consisting of a Focused-Ion-Beam (FIB) column and an SEM column on the same platform, where a thin lamella was mounted on a support Cu-grid.

\subsection{DTE testing}

DTE testing was performed in the light gas gun facility at the University of Cassino and Southern Lazio. The apparatus is a single stage gas gun $3.50 \mathrm{~m}$ long with a $7.62 \mathrm{~mm}$ bore. The maximum pressure of the reservoir was 300 bar. The gun is fired bursting a Mylar ${ }^{\circledR}$ disk by means of a thermo-resistance. This solution allows full control on the firing pressure, and 
consequently on the achievable velocity, at which the test is performed. Projectile dimensions are given in Figure 1. Before each test, the gun and the die were cleaned in order to minimize friction. All tests were performed in vacuum and ejected fragments were soft recovered using a ballistic gel block. The projectile used in this study was designed to have the same mass as the spherical bullet used in [9]. Compared with the spherical shape, the proposed geometry is easier to machine and allows checking of the projectile alignment at impact. In fact, the rear section of the fragment in the die remains perpendicular to the symmetry axis if the projectile is aligned at the time of the impact with the die. A cork sabot was used to minimize pressure losses.

The die has the same geometric characteristics of that used by Gray III et al. [9]. Size and dimensions are given in Figure 2. In order to avoid propelling gas overpressure after the impact in the die, recoil compensator located at the end of the gun before the die, was used. The velocity of the projectile was measured just before entering the die using two laser beam photodiodes. The dynamic extrusion process was recorded using high speed video camera at 70.000 frames per second. This provides a mean to estimate the muzzle velocity and to obtain time resolved shape of the jet.

Two series of tests were performed at 350 and $400 \mathrm{~m} \mathrm{~s}^{-1}$, respectively. At higher velocity, the extruded jet produced three fragments (overall elongation $\sim 35 \mathrm{~mm}$ ) while at lower velocity only one fragment (overall elongation $\sim 23.5 \mathrm{~mm}$ ) was extruded. The fragment that remains in the die is subjected to strain ranging from almost no deformation in the rear section to very high strain in the front necked section. This fragment is a good candidate to correlate microstructure evolution with strain and to use this information to support constitutive model verification. Therefore, this segment is the focus throughout the present investigation.

\section{NUMERICAL SIMULATION}

To probe material response at large strain finite element simulation was used. The DTE impact test at $400 \mathrm{~m} \mathrm{~s}^{-1}$ was simulated with the implicit FEM code MSC MARC v2013. Since the problem is axisymmetric and symmetries are preserved during the deformation and fragmentation process, a 2D model was developed. The projectile and the die were simulated as deformable bodies in contact using fully integrated (four Gauss points) four node isoparametric elements with bilinear displacement functions. The analysis was carried out using large displacement, Lagrangian updating and finite strain formulation. The dynamic transient was simulated using single-step Humbolt operator, which is second order accurate, asymptotically 
annihilating and it has the same unconditional stability as the Newmark-beta operator. Coupled thermo-mechanical analysis, taking into account the conversion of the plastic work to heat, was performed. The role and the effect of heat exchange between the projectile and the die was also analyzed. Since the projectile undergoes very large deformation, global re-meshing was used to avoid excessive element distortion and consequent loss of accuracy in the results. Re-meshing parameters were selected to preserve both element average size and aspect ratio during the entire analysis avoiding artificial stress wave reflections caused by the difference in the mechanical impedance between adjacent elements of different sizes.

Friction between the projectile and the die unfortunately represents an unknown that can significantly affect the computational results. Since it cannot be measured experimentally, this presents an unresolved disconnection between the numerical simulation and experiment. In addition, friction models available in FEM codes were not developed to reproduce the conditions occurring in dynamic impacts. Therefore, the contact between the projectile and the die was assumed frictionless at this stage.

Two strength models were considered to simulate OFHC copper response under dynamic deformation: Johnson-Cook and Zerilli-Armstrong. The first is a pure phenomenological model while the latter is based on dislocation mechanics and accounts for the material crystal structure. In their original formulations, for both models, stress does not saturate at large strain. Since in the DTE large strain are expected, a modified version of each model as used. In the JohnsonCook, hereafter $\mathrm{mJ}$-C, the first term was replaced by a two-terms Voce type law,

$$
\sigma=f\left(\epsilon_{p}\right)\left(1+C \ln \frac{\dot{\epsilon}}{\dot{\epsilon}_{0}}\right)\left(1-T^{* m}\right)
$$

where,

$$
f\left(\epsilon_{p}\right)=\sigma_{y}^{0}+B_{1}\left(1-\exp \left\{-\frac{\epsilon_{p}}{t_{1}}\right\}\right)+B_{2}\left(1-\exp \left\{-\frac{\epsilon_{p}}{t_{2}}\right\}\right)
$$

While for the Zerilli-Armstrong, hereafter mZ-A, the modified version proposed in [18] was used,

$$
\sigma=C_{0}+C_{2}\left(\epsilon_{r}\left[1-\exp \left\{-\frac{\epsilon_{p}}{\epsilon_{r}}\right\}\right]\right)^{1 / 2} \exp \left\{-C_{3} T+C_{4} T \ln \epsilon_{p}\right\}
$$

For the mZ-A model material parameters were taken from the literature, while for the mJ-C parameters were identified based on quasi-static and Hopkinson pressure bar traction and compression tests [19]. 
At strain rates larger than $10^{3}-10^{4} \mathrm{~s}^{-1}$ copper shows a transition from thermal activation controlled deformation to dislocation drag controlled deformation [20]. In this regime, materials show an increased strain-rate sensitivity usually attributed to the electron and phonon-drag effects on the mobile dislocations [21, 22]. Both J-C and Z-A models, in their original formulations, do not consider this transition. Therefore, for DTE test simulation purpose, the strain rate sensitivity parameter in both models was adjusted to reproduce the shape and size of the fragments for the $400 \mathrm{~m} \mathrm{~s}^{-1}$ DTE test. The set of calibrated parameters is summarized in Table 1.

In DTE test of OFHC copper, extruded jet fragmentation occurred by strain localization sustained by thermal softening, which reduces the neck section to a point. This behavior could be accurately reproduced using global re-meshing. Consequently, no damage model was used and a simple prescribed maximum strain criterion for fragment separation was used.

Once calibrated, numerical simulation was used to extract plastic strain, strain rate and temperature histories at selected locations to correlate with EBSD examination results.

\section{Results}

\subsection{Mechanical response}

Numerical simulation of DTE test at $400 \mathrm{~m} \mathrm{~s}^{-1}$ has been repeated with the two selected strength models. These formulations have different derivation and have been used to verify that calculated strain, strain rate and temperature histories were not specific of the constitutive model used. Model parameters have been calibrated in order to reproduce the same plastic flow curve for the reference strain rate of $1.0 \mathrm{~s}^{-1}$ and the strain rate sensitivity parameters were adjusted in order to match fragment shape and size observed in the experiment. In Figure 3 the comparison of the predicted fragments size and shape for $400 \mathrm{~m} \mathrm{~s}^{-1}$ fired test is shown. Both models predicted fairly well the overall number of fragments, and the size of all fragment except for the second one. For this, both models predicted an excessive thinning. Another difference is that, for the first fragment, both models predicted a more pronounced necking than that observed in the experiment. The third and fourth fragments are fairly well predicted by both models. The mJ-H seems to provide better agreement with experimental finding than the mZ-A.

The attention of present investigation was focused on the analysis of the fragment that remains in the die. Five locations along the symmetry axis, hereafter indicated as sample point 1 to 5, were selected as shown in Figure 4. In Figure 5 the comparison of stress and strain rate vs 
plastic strain histories, for sample point 1 and 5, calculated with $\mathrm{mJ}-\mathrm{C}$ and $\mathrm{mZ}-\mathrm{A}$ is shown. Both strength models gave similar results. The mZ-A resulted always in larger plastic strain than $\mathrm{mJ}-\mathrm{C}$. Similar agreement was found for all other investigated sample points (regions).

In Figure 6 the evolution of von Mises equivalent stress, strain rate and temperature as a function plastic strain for all five sample points is shown. For each sample point, the strain rate is almost constant during the entire deformation process, increasing from $3 \cdot 10^{4} \mathrm{~s}^{-1}$ to $3 \cdot 10^{5} \mathrm{~s}^{-1}$ for sample point 1 to 5 , respectively.

The Zener-Hollomon parameter is a common way to characterize non-isothermal, nonconstant strain rate deformation. Therefore, the parameter $Z$, which is defined as,

$$
Z=\dot{\epsilon} \exp \left\{\frac{Q}{R T}\right\}
$$

was selected to compare deformation paths at selected sample points. In Eqn. (4), $R$ is the ideal gas constant, $T$ is the temperature in $[\mathrm{K}]$, and $Q$ is the apparent activation energy of the deformation process. Simulation results showed that temperature at different sample points varies approximately from $300 \mathrm{~K}$ up to $1000 \mathrm{~K}$ during the dynamic extrusion. Over such large temperature range, the apparent activation energy is not constant and its variation with temperature should be considered. For high purity copper, the following phenomenological expression is proposed:

$$
Q=Q_{l} \tanh \left(A^{\prime}\left[\frac{T}{T_{m}}\right]^{\beta}\right)
$$

For copper, activation energy data [23-25] - collected by Freed et al. [26] - as a function of temperature are shown in Figure 7 together with Eqn. (5) fitted curve. Therefore, combining eqn. (4) with eqn. (5), the following expression for the parameter $Z$, accounting for temperature dependence of apparent activation energy, can be obtained:

$$
Z=\dot{\epsilon} \exp \left\{\frac{Q_{l} \tanh \left(A^{\prime}\left[T / T_{m}\right]^{\beta}\right)}{R T}\right\}
$$

where $Q_{l}$ is the saturation value equal in value to the activation energy for self-diffusion $\left(Q_{l}=210\right.$ $\mathrm{kJ} / \mathrm{mol}$ ), $A^{\prime}$ and $\beta$ are material constants and $T_{m}$ is the melting temperature.

The evolution of $\log (Z)$, as given in eqn. (6), as a function of plastic strain for the selected sample points is shown in Figure 8 . To be noted that $\log (Z)$ vs plastic strain paths fall on the 
same curve indicating that the deformation history is the same for all sample points. The parameter $\log (Z)$ decreases, with increasing plastic strain, from 30 asymptotically down to 15 .

The parameter $Z$ can also be used to predict the condition for dynamic recrystallization (DRX) to occur. In plastically deformed material, the critical strain $\varepsilon_{c r}$ at which DRX initiates depends on the temperature, strain rate, initial grain size and alloying. The phenomenological relationship that provides the critical strain as a function of $Z$ has the following form,

$$
\epsilon_{c r}=K_{\epsilon} Z^{m}
$$

where $K_{\varepsilon}$ is a function of the initial grain size, $d_{0}$, and alloy content. In the literature, $K_{\varepsilon}$ is assumed to be a power law of the initial grain size. However, in copper, the critical strain for DRX increases with the initial grain size showing a rapid increase for smaller initial grain size and eventually reaching a saturation for large initial grain size $[27,28]$. The authors found that a Voce type law provides a much better description of the evolution of the constant $K_{\varepsilon}$ as a function of the initial grain size, limiting the critical strain for DRX at very large values of the initial grain size,

$$
K_{\epsilon}\left(d_{0}\right)=\bar{K}_{\epsilon}\left(1-\exp \left\{-\frac{d_{0}}{d_{0}^{*}}\right\}\right)
$$

where $d_{0}^{*}$ is a material constant, equal to $22.4 \mu \mathrm{m}$ for OFHC copper, and $\bar{K}_{\varepsilon}$ is a function of the purity of the metal, equal to $1.89 \mathrm{E}-03$ for 99.98 commercially pure copper [29].

In Figure 8, the Eqn. (7) is plotted for 99.99\% OFHC copper with an initial grain size of $340 \mu \mathrm{m}$ and compared with experimental data of Gao et al. [30] obtained at low strain rate. In the same plot, the solution for $15 \mu \mathrm{m}$, which is the average grain size for the material using in the present investigation, is also drawn. Here, the condition for DRX is predicted to occur in the DTE test at $230 \%$ of plastic strain, which is reached and exceeded at sample point 3 to 5 .

\subsection{Deformation structure}

To follow the evolution of the structure of the deforming material, five regions corresponding to the previously discussed sample points, as well as the annealed material, were investigated using EBSD. The microstructure of the as-annealed material is shown in Figure 9. The inverse pole figure (IPF) map in Figure 9(a) indicates a random texture of the microstructure, which contains numerous annealing twins. The grain size was measured to be 14 $\mu \mathrm{m}$ by the linear intercept method using the EBSD data. This measure includes the twin boundaries, as they are the dominant grain boundary type. Excluding the twin boundaries 
results in a measured grain size of $47 \mu \mathrm{m}$. The pole figures (PF) in Figure 9(b) confirms the random starting texture.

The evolution of the microstructure during dynamic tensile extrusion is shown in Figure 10 , where the grains are outlined by high-angle boundaries ( $\mathrm{HAB}, \theta>10^{\circ}$ ) in black, with the special case of twins $\left(\Sigma 3, \theta=60^{\circ}\right)$ shown in red. Also included are low-angle boundaries (LAB, $2<\theta<10^{\circ}$ ). Figure 11 shows the distribution of misorientation angles while the relative frequencies of different types of boundaries (HAB, LAB, $\Sigma 3$ and $\Sigma 9+\Sigma 27(\mathrm{a}, \mathrm{b}))$ are shown in Figure 12. The annealed microstructure is clearly dominated by twin boundaries, and there is also a noticeable fraction of other CSL boundaries. The presence of $\Sigma 9$ and $\Sigma 27$ boundaries is indicated in Figure 11(b).

In region 1 , corresponding to a plastic strain of 1.2 , the amount of twin boundaries is drastically reduced, and the other CSL boundaries have disappeared. A large increase in the LABs can be seen as a result of the plastic deformation. The density of LABs in different grains is indicative of stored (geometrically necessary) dislocations. It can be clearly seen from Figure 10 (in particular in (b) and (c)) that the distribution is not homogeneous. Whereas most of the grains have a high homogeneous intra-grain LAB distribution, others exhibit very low density of LABs or local regions with high LAB density in otherwise almost LAB free grains. In the latter grains, the majority of the LABs are generally found closer to the grain boundaries, especially at deformations up to a plastic strain of 1.9 (sample point 2). With increasing deformation, up to region 4 (plastic strain of 3.1), the fraction of LABs decreases in favor of general HABs. This is indicative of a progressive grain subdivision and fragmentation during deformation. The fraction of twin boundaries remains roughly constant and other CSL boundaries are still absent.

During the course of deformation the grains become progressively elongated in the extrusion direction with increasing strain. The conversion of LABs to HABs can also be clearly seen. In region 4, recrystallized grains can be seen as equiaxed, or close to equiaxed, crystals without any deformation induced LABs. A small number of such recrystallized grains also exist in region 3 . The degree of recrystallization increases significantly with deformation beyond region 4, as can be seen in region 5 (plastic strain of 5.4). Here the majority of the microstructure consists of recrystallized grains free of internal deformation induced LABs. The fraction of twin boundaries has increased to around $20 \%$, and the other CSL boundaries have also reappeared. The ratio of $(\Sigma 9+\Sigma 27): \Sigma 3$ is around 0.2 in both the annealed microstructure (0.19) and sample point 5 (0.23). 


\subsection{Grain size}

The grain size (here taken as the diameter of a circle of the same area as the grain) for the different investigated regions, including twin boundaries, were extracted from the data sets by letting the software perform corrections for edge intersection. During this procedure, the grain size is calculated based on a minimum misorientation angle of $10^{\circ}$. Then the size of each grain is multiplied by two if it intersects one edge of the EBSD map, and by four if it intersects two edges. Figure 13 shows the grain size histograms for the different regions. A general shift of the distribution towards smaller sizes can be seen with increasing strain. Additionally, a few extreme points can be seen, which do not fit the remaining distribution. These outliers are a result of single very large grains intersecting two edges, the already large size thus being quadrupled. Another way of representing the grain size distribution, instead of by number fraction as in Figure 13, is by area fraction. The area fraction distribution is shown in Figure 14, where much more even distributions can be seen. The average grain size based on the two types of representations (excluding the outliers) is shown in Figure 15. Both measures follow the same trend of decreasing and saturating size with increasing strain, but the decrease is more rapid for the average based on number fraction. Also included (for the number fraction data) is the effect of choosing different grain size thresholds for inclusion in the data. The lower limit corresponds to inclusion of all identified grains, even if they are only one pixel in size. The upper limit is based on a minimum of ten pixels in a grain, and the average corresponds to a minimum of 5 pixels. For the average based on area fractions, the effect of selected threshold is insignificant. While the effect is limited also for the number fraction based average in terms of absolute values (deviations from the 5 pixel average of between 0.9 and $5 \mu \mathrm{m}$ ), it is large in relative terms (deviations between 13 and $41 \%$ ). Nevertheless, the trend is the same independent of selected threshold.

\subsection{Texture}

The elongation in the extrusion direction is accompanied by a strong texture development, as shown in Figure 16. The corresponding IPF maps are shown in Figure 17, indicating the development of a dual $<001>+<111>$ fibre texture. The strengths of the different texture components are shown in Figure 18, based on integration over $15^{\circ}$ from the ideal orientations. The choice of $15^{\circ}$ was made based on the observed distribution of deviations from the ideal $<001>$ and <111> orientations. Whereas the components were initially roughly equal in strength, the fraction of the $<111>$ component increases more rapidly. Both components appear 
to saturate with strain, the $<001>$ component earlier (at a strain of 2) than the $<111>$ component (at a strain of 2.5). There is a clear difference at the largest strain, where the $<111>$ component fraction is reduced from 65 to around $20 \%$, while the <001> component increases from 30 to 37 $\%$. The reduction in the $<111>$ fraction is a result of the extensive recrystallization, where $<111>$ oriented regions are preferentially replaced by grains with $<001>$ orientation. The remaining unrecrystallized regions primarily consist of $<001>$ grains, although some few remaining $<111>$ oriented grains also exist. Also included in Figure 18 is the development of the fraction of recrystallized grains.

\section{Discussion}

\subsection{Deformation substructure}

In the present investigation, an inhomogeneous distribution of LABs during deformation, both on inter and intra-granular levels, was observed. While the $<001>$ orientation is softer compared to the $<111>$ orientation (ideal Schmid factor of 0.408 compared to 0.272 ) and should deform more readily, signs of the opposite situation can be seen in Figure 10. It has been shown both experimentally [31] and by crystal plasticity simulations [32] that the surrounding grains can have a large influence on the deformation response. Soft grains surrounded by harder grains have been demonstrated to exhibit a lower number of geometrically necessary dislocations than their harder neighbors [31]. The proposed explanation is the much lower degree of hardening expected in grains oriented for easy slip, which result in storage of dislocations primarily at the grain boundaries [31]. In comparison, the deformation of harder grains will produce a higher density of dislocations, which are stored more homogenously in the grain interior. Figure 19 shows a TEM micrograph from region 4, obtained at the boundary between two grains close to the $<111>$ and $<001>$ orientations. While the $<111>$ grain exhibits a cell structure, the $<001>$ oriented grain contains very elongated cells, or microbands. The difference in dislocation structure between the two texture components was confirmed through observation at several locations. Gray III et al. [9] observed both microbands and dislocation cells in DTE deformed copper, but here we can also conclude that the structures observed depend on the orientation of the investigated grain(s). Clearly, this orientation-dependent substructure development is of outmost interest for the understanding of the operative deformation mechanisms and grain interactions during straining. 
The twins observed in region 5 are believed to be annealing twins formed during growth of the recrystallized grains. This is indicated both by the similarities with the annealed structure in terms of grain boundary types etc., but also by the absence of plastic deformation in the recrystallized structure. In regions 3 and 4, on the other hand, the twins could be suspected to be a result of deformation induced twining. Twinning has been shown to be a potential contributor to the deformation of copper during dynamic conditions, and can occur at much less extreme conditions than predicted by models [33]. It should also be noted that twinning under dynamic conditions have been shown to be an orientation dependent phenomenon, where copper specimens deformed in compression by dynamic plastic deformation primarily showed twinning in grains with orientations near $<001>$, but not closer to the $<101>$ corner [34]. The fact that twins at intermediate strains in the present investigation appeared to be primarily associated with <001> textured regions could be interpreted as support of this. However, as the microstructure is so dominated by the $<001>$ and $<111>$ fibres, the number of grain boundaries with a misorientation close to the ideal $54.7^{\circ}$ will be high. Given that the grains are not ideally oriented, there will likely be a quite large fraction in the angular range of $58-62^{\circ}$, which was here used to identify the $\Sigma 3$ boundaries in Figure 10. A considerable portion of the observed $\Sigma 3$ boundaries is therefore probably not formed through mechanical twinning, but through dislocation slip-mediated grain rotation. This was also verified by detailed analysis of a number of individual boundaries. No signs of deformation twins similar to those observed in coarse grained OFHC copper in [14] could be found in the present case. All in all, the observations indicate that deformation twinning does not contribute significantly to the deformation of copper under DTE conditions.

\subsection{Texture development}

Qualitative investigations of the texture in copper during DTE have been reported by Grey III et al. [9] and Park et al. [14], but no quantifications of the texture component strengths were provided. More quantitative results have been reported during quasi-static tensile testing up to strains of 1.0 [35]. A number of studies are available on the texture resulting from wire drawing, e.g. [36-39], but usually with limited quantitative information. All studies show the development of a strong dual $<001>+<111>$ fibre texture, although the texture is not homogeneous throughout the wire in the case of drawing as a result of the friction induced shearing at the surface. Figure 20 shows compilations of the available quantitative data found in the literature in comparison to the present results. As the absolute values of the measured area/volume fractions 
depend on the chosen range of angles for integration, the comparison is not always straightforward. In most cases, no information on how the texture component strengths were calculated was provided. In [35] it was mentioned that it is normally calculated by integration over 15 or $20^{\circ}$. In this study, $15^{\circ}$ was found to be the best choice, but in Figure 20 results using both $15^{\circ}$ (open data points) and $20^{\circ}$ (solid data points) are included for comparison. The difference between the two is largest at lower strains, where the texture is less pronounced and the uniform background makes a larger contribution. Clearly, the fraction of the <001> fibre agrees rather well with the literature, if the recrystallization at large strains is disregarded. The fraction of the $<111>$ fibre (and thereby also the sum $<001>+<111>$ ) is higher in the present case. Also the development of the relative strengths (ratio $<001>/<111>$ ) is qualitatively similar in development.

The primary comparison that can be made is with the quasi-static results from [35]. The qualitative development of the texture components is similar, but, as mentioned, the $<111>$ (and $<001>+<111>$ ) fraction is higher in the present case. Many factors can be expected to influence the development of the texture. It was suggested [40] and experimentally verified [41] that the texture development during rolling of $\mathrm{Cu}-5 \% \mathrm{Zn}$ is characterized by an activation energy, which was suggested to be the activation energy for cross-slip, resulting in a strain rate dependence of the texture. In OFHC copper, the effect of strain rate on the texture has been demonstrated using both shear compression specimens [42] and split Hopkinson pressure bar [43]. The texture index and the <101> component increased at strain rates above $100 \mathrm{~s}^{-1}$ [43]. Also in [42] a change in the relative strengths of different components with increasing strain rate could be observed, primarily leading to a partial replacement of the $<001>$ orientation with a $<101>$ shear component, whereas the $<111>$ component remained unaffected. The proposed reason was the increase in strain hardening rate at higher strain rates. While the modes of deformation employed are not representative of the present study, the results indicate that the effects of strain rate cannot be neglected when making comparisons.

Other potentially important factors are the initial grain size and starting texture of the tested material. Naaman et al. [35] observed an increased <111> component, and a decreased $<001>$ component with increasing grain size (from 23 to $125 \mu \mathrm{m}$ ) in copper at strains up to 0.3. However, the two materials also differed in starting texture (both rather weak dual $<001>+<111>$ texture, but with different relative strengths), and it was concluded that the extent of the contributions from texture and grain size were difficult to assess due to complicated interactions. It was, however, clear that the effect of starting texture is much larger 
in tension than in compression. Simulations presented in [35] based on a random starting texture, which was evolved based on a gradual change-over from the full constraints to the relaxed constraint Taylor model, reached $<001>+<111>$ fractions similar to those observed in the present case at a strain of 1.0 (see Figure 18(a)).

To investigate the relative effect of starting texture on the subsequent fractions of texture components, the crystal plasticity package CPFEM [44], developed for use as a UMAT routine to the ABAQUS ${ }^{\circledR}$ finite element software, was used. A single element represents an arbitrary number of discrete orientations, which are all assumed to undergo the same strain as prescribed macroscopically (Taylor assumption). The starting textures were created using the MTEX software [45]. By defining different synthetic orientation distribution functions (ODFs), data sets with 1000 discrete orientations (grains) were generated as input. Three test cases were used: (i) completely random texture; (ii) "weak" $<001>+<111>$ fibre texture (which was tuned to have a IPF as similar as possible to the similar to the starting material in [35]); and (iii) a "strong" texture, which was similar to the "weak" case but more pronounced (texture index 1.47 compared to 1.03). Parameters for the crystal plasticity model for pure copper were taken from [46]. All simulations were performed in uni-axial tension to a total strain of 5.0, with intermediate textures being exported at regular intervals. The resulting textures were analyzed using MTEX, where the volume fractions of $<001>$ and $<111>$ fibres were calculated by integration over $15^{\circ 1}$.

Results presented in Figure 21 clearly show that an increasing strength of the initial dual fibre texture results in a weaker $<111>$ component, and a stronger $<001>$ component. These results correlate well with the observations above, where the volume fractions of <111> obtained from the present random starting texture (as well as the simulations with random starting texture presented in [35] were higher than observed in the weakly texture material in [35], whereas the $<001>$ fractions were similar. The trends point to a significant influence of starting texture on the subsequent development, even if the dominating initial texture components are similar to the deformation texture. While the calculated volume fractions are much higher than the experimentally observed levels, in particular the $<111>$ fraction, and the rate of development with strain is more rapid, more realistic simulation results could be obtained by using e.g. visco-plastic self-consistent models [47, 48], especially considering co-

${ }^{1}$ If $20^{\circ}$ was used instead, the resulting initial fractions for the weak starting texture corresponded well to those seen in [31], which was the intention. Also, for integration over $20^{\circ}$ the texture evolution of the random starting texture was identical to the model result presented in [31]. 
rotation effects, which have been proven to give very good predictions for pure Cu under large strain deformation [49], or relaxed constraints models. The present simulations also neglect any strain rate effects on the texture development. However, at this stage the purpose of the simulations was only to study qualitative trends resulting from changes in the starting texture, and hence the CPFEM results were considered sufficient.

During DTE, it has been observed that a smaller grain size (65 $\mu \mathrm{m}$, "weak initial texture”) produced equally strong $<001>$ and $<111>$ fibres, whereas larger grain sizes (118 and $185 \mu \mathrm{m}$ ) resulted in a dominating $<111>$ fibre texture [9]. However, in [9] the details on the microstructural observations were not included. Park et al. [14] also observed the dual $<001>+<111>$ texture during DTE of coarse-grained OFHC copper with a grain size of $120 \mu \mathrm{m}$ (random starting texture). No investigation of the segment left in the die was presented, but the extruded segments showed "strong" $<111>$ and "moderate" <001> components.

Based on these observations, the random starting texture and smaller grain size in the present investigation could be expected to result in a dominating $<111>$ texture, and a stronger total texture due to both starting texture and the high strain rate. This is in general agreement with observations, although a parametric study of the effects of grain size, strain rate and initial texture would be necessary to draw any firm conclusions.

The appearance of a $<001>$ recrystallization texture from the deformed $<111>$ fibre can be understood in terms of the strain-energy-release maximization (SERM) model [50, 51]. The absolute maximum principle stress in $<111>$ oriented crystals will be in the $<111>$ direction. During formation of new grains, the released strain energy will be maximized if the crystal direction with minimum elastic modulus ( $<001>$ in copper) is aligned with the axis of principle stress. Therefore, the deformed $<111>$ grains will tend to be replaced by recrystallized $<001>$ grains. For the same reason, $<001>$ regions will be replaced by $<001>$ oriented grains during recrystallization.

\subsection{Conditions for recrystallization}

In previous investigations of DTE of coarse-grained $\mathrm{OFCH} \mathrm{Cu}[9,14]$, no evidence of recrystallization was reported. Park et al. [14] used the same spherical bullet as in [9] but a different die geometry with larger outlet opening and higher impact velocity. Performing a simulation with the same set-up as in [14] and current material model, it was found that the strain history, given in terms of $\log (Z)$ parameter versus plastic strain, for the points along the axis of the fragment in the die are very similar to those reported in Figure 8 for current DTE test configuration. The near tip region investigated in [14] is approximately $3.3 \mathrm{~mm}$ from the end of 
the fragment, which corresponds to a point in between region 3 and 4 in our case. The exact location where observations are performed and compared is important since it significantly influence the results. The deformed structure, close to the tip of the segment left in the die, was illustrated by optical micrographs in [14]. No texture measurements were reported for this segment after deformation. It is possible that the early stages of DRX had indeed occurred, but the very small recrystallized grains (as in regions 3 and 4 in Figure 4) was not resolvable. In [14], texture measurements were performed in the extruded fragments with no indications of recrystallization. The early stages of recrystallization are not clearly distinguishable in the IPF and misorientation distributions, without more detailed analysis. In the present study, extruded fragments were not examined and therefore it is not possible to establish and compare the state of recrystallization. Failure by localized shear band, at the tip of the fragment in the die, was not reported neither in other investigations [9] nor observed in this work. It may be a consequence of the experimental set-up (i.e. projectile misalignment at the impact and subsequent contact in the die channel). The initial grain size of the annealed copper tested in [14] $(120 \mu \mathrm{m})$ differs from that measured in the present study $(15 \mu \mathrm{m})$, and this also has an influence on the recrystallization conditions as predicted by Eqn. (7) and (8) and shown in Figure 8. In summary, it is not clear if DRX did not occurred in the DTE tests in [14] due to experimental reasons, or if it did so only to the minor extent (in the investigated regions) that it was not resolved in the analysis. In Gray III et al. [9], much less information on the microstructure investigations were provided, and no high-resolution SEM or EBSD images are shown, which makes difficult to perform direct comparisons. No signs of recrystallization are mentioned in the paper, and TEM images from the extruded segments showed deformed microstructures with subgrains and microbands, similar to the present case.

Numerical simulations, presented in this work, have shown that the use of the ZenerHollomon parameter is an efficient way to characterize the deformation history at selected locations. The parameter $Z$ allows one to compare complex deformation path involving changes in the strain rate and temperature. In addition, considering the temperature dependence of the apparent activation energy allows compensation for different mechanisms governing the deformation process. The major finding, shown in Figure 8, is that post-mortem investigation of material microstructure at different locations along the axis of the segment in the die is equivalent to look at the microstructure evolution in situ, at different time instants, during the dynamic deformation process. Equation (7) provides a mean to estimate the condition for initiating DRX and, as shown in Figure 8, the location at which it can occur. As predicted by Eqn. 
(8), for the same deformation process (i.e. same $\log (Z)$ vs plastic strain path), the larger the initial grain size the larger is the critical plastic strain for DRX to occur. In [9] the initial grain size ranged from 65 to $185 \mu \mathrm{m}$. Although the bullet geometry differs from that used in this work (but same projectile mass, velocity and die geometry), it was verified that the $\log (Z)$ vs plastic strain path is the same as that shown in Figure 8. According to present analysis results, DRX is predicted to occur for all three initial grain sizes, but with lower extent for larger grain size, at region $4(<1.75 \mathrm{~mm}$ from the tip of the segment) for plastic strain exceeding $\sim 2.5$ and $\log (Z)$ 17. In general, failure strain decreases with reductions of the initial grain size. However, the two effects of grain refinement and lowering of dislocation and twin densities during DRX, both benefit the ductility during deformation and this may explain the results observed by Gray III et al. [9].

In DTE test, the length of the extruded jet can be used to have an estimate of material ductility. Park et al. [14] defined the ductility of the jet as the sum of the axial ductility of each fragment with respect to the initial sample diameter. However, it is known that for given projectile mass and die geometry, the initial velocity has an influence on the resulting overall length of the jet [11]. Therefore, it was decided to define the velocity compensated average engineering strain of the jet as the ratio of the sum of all fragments lengths normalized by the initial projectile length (because engineering strain is not additive) normalized by the impact velocity:

$$
\epsilon^{*}=\frac{1}{v} \frac{\sum_{i=1}^{N} l_{i}-l_{0}}{l_{0}}
$$

where $l_{i}$ is the longitudinal length of the $i^{\text {th }}$ fragment, $l_{0}$ is the initial bullet length and $v$ is the impact velocity. In Figure 22, the velocity compensated ductility as a function of the initial grain is shown. Present result is in very good agreement with the trend shown by data provided by Gray III et al. [9]. Also the datum relative to coarse grain copper, taken from by Park et al. [14], is now in agreement with the observed trend with the exception of data of ultra-fine grain copper, for which a different behavior is expected.

\subsection{Recrystallization mechanism}

Two types of DRX are generally considered: continuous (cDRX) and discontinuous (dDRX) recrystallization [52]. The former is based on progressive rotation of sub-grains to create HABs, or in some cases to the changes in grain geometry during straining (usually termed geometric 
dynamic recrystallization). The mechanism of $\mathrm{dDRX}$ is different, in that it occurs through nucleation of new grains at old HABs, followed by growth of the recrystallized grain into the deformed matrix. During cDRX, both due to geometric reasons and sub-grain rotation, the development of a texture markedly different from the deformed state is not expected. In contrast, Figure 17 clearly shows a $<001>$ texture component replacing the $<111>$ due to recrystallization, indicating that $\mathrm{dDRX}$ is the dominating mechanism. This is expected in metals with low-to-medium stacking fault energy, such as copper.

In the present case, relatively large recrystallized grains in region 5 , at least large compared to the nuclei seen in regions 3 and 4, were observed. The average grain size in region 5 was in the order of $5 \mu \mathrm{m}$, but some of the recrystallized grains were as large as $50 \mu \mathrm{m}$. To assess the feasibility of $\mathrm{dDRX}$ as the operative mechanism, the model for grain growth during $\mathrm{dDRX}$ used by Cram et al. [53, $54]$ is employed. In this model, the velocity of a grain boundary, $v g b$, at temperature $T$ is described by

$$
v_{G B}=\beta \frac{\delta \Omega_{m} \mu \Delta \rho}{2 R T} D_{0, G B} \exp \left\{-\frac{Q_{G B}}{R T}\right\}
$$

where $\beta$ is the effective fraction of the Turnbull estimate of grain boundary mobility [55], $\delta$ is the grain boundary width, $\Omega_{m}$ is the molar volume, $\mu$ is the shear modulus, $R$ is the gas constant, $D_{0, G B}$ and $Q_{G B}$ are the pre-exponential and activation energy, respectively, for grain boundary diffusion, and $\Delta \rho$ is the difference in dislocation density between deformed and recrystallized grains. For a non-isothermal process, the size of an assumed spherical grain is found by integrating Eqn. (10) over the thermal history

$$
d(t)=d_{i}+2 \int_{t=0}^{t} v_{G B} d t
$$

As in $[53,54]$ the initial grain size, $d_{i}$, is based on an estimated critical size for the BaileyHirsch grain boundary bulging mechanism [56],

$$
d_{i}=\frac{8 \gamma}{\mu \rho b^{2}}
$$

The final equation for the grain size evolution, assuming a constant dislocation density, then becomes

$$
d(t)=d_{i}+2 \beta \frac{\delta \Omega_{m} D_{0, G B} \Delta \rho}{2 R T} \int_{t=0}^{t} \frac{\mu[T(t)]}{T(t)} \exp \left\{-\frac{Q_{G B}}{R T}\right\} d t
$$


Here the temperature dependence of the molar volume as well as the effects of high dynamic pressure on molar volume and shear modulus are neglected. The values of the parameters for pure copper are summarized in Table 2. The dislocation density in copper during quasi-static severe plastic deformation have been measured in the range $1.5 \cdot 10^{14}-3 \cdot 10^{16} \mathrm{~m}^{-2}$ at large strains $[57,58]$. Assuming that the recrystallized grains are dislocation free, and that the dislocation density in the deformed grains is higher end of the above range during dynamic conditions, we used $\Delta \rho=10^{15}$ and $10^{16} \mathrm{~m}^{-2}$ in our simulations. The critical nucleus size is estimated to around 2 and $0.2 \mu \mathrm{m}$ for dislocation densities of $10^{15}$ and $10^{16} \mathrm{~m}^{-2}$, respectively, using Eqn. (12). Integrating Eqn. (13) over the part of the thermal history exceeding $0.4 T_{m}$, which is usually taken as an approximate lower temperature limit for DRX, results in grain growth of only fractions of a $\mu \mathrm{m}$. It is therefore not likely that the observed grain growth occurred during deformation. The most probable course of events in the present case is that the dDRX occurred during deformation, but grain growth took place after the deformation stopped, while the temperature was still high enough during cooling of the specimen inside the die. This phenomenon is usually termed meta-dynamic recrystallization [59], mDRX, and typically results in a microstructure consisting of a mix of small and large recrystallized grains and deformed unrecrystallized regions [52], similar to the present case. To verify this hypothesis, a second simulation was performed, including the part of the test after the deformation stopped. The temperature redistribution and cooling were simulated by considering heat transfer through conduction in both specimen and die, radiation and natural convection. The thermal conductivity, emissivity and specific heat of pure copper and tool steel were used for the specimen and die, respectively. The natural heat convection coefficient was taken to be $10 \mathrm{~W} \mathrm{~m}^{-2}$ $\mathrm{K}^{-1}$, and the environment was modeled as a heat sink with temperature $298 \mathrm{~K}$. The resulting temperature and grain growth simulations for $\Delta \rho=10^{15}$ and $10^{16} \mathrm{~m}^{-2}$ can be seen in Figure 23. Depending on the assumed dislocation density, the final grain size ends up in the range of 5 to 32 $\mu \mathrm{m}$, which is consistent with the experimental observations. It should also be noted that since in principle all grain growth occurs after the deformation has stopped, there is no subsequent deformation of the recrystallized grains, which would slow down the grain growth. This supports the use of a constant value of $\Delta \rho$ in the calculations. The growth rate can therefore be expected to be higher during this type of mDRX compared to typical dDRX during testing under e.g. hot working conditions where the continued deformation of the recrystallized microstructure continuously reduces the driving force for grain growth. 
It should be noted that the grain growth simulations are of course extremely sensitive to the input temperature and activation energy due to the exponential expression. The simulation of the cooling is also inherently approximate, since the perfect thermal contact between the specimen and die was assumed. Furthermore, simulations indicate that at the end of the deformation the reflected shock wave will eject the conical piece from the die. This is in agreement with observations when removing the pieces from the die after the experiment, where the conical specimen was found lying free inside the die, close to the nozzle. This will of course result in boundary conditions far from the idealized case used in the simulations. However, both the addition an interface heat transfer coefficient and boundary conditions corresponding to a "free" specimen would result in slower cooling rates. Thus, the conclusions regarding the feasibility of the occurring grain growth during cooling still hold, provided that the activation energy is not significantly lower than the value used in the present case.

Recrystallization in OFHC copper during dynamic tensile deformation clearly requires further investigations, utilizing high-resolution characterization methods capable of resolving the very early stages of nucleation. Based on the present tests such a dedicated investigation of the mechanisms of recrystallization will be undertaken, and presented in as a separate study.

\section{CONCLUSIONS}

In this work, the evolution of microstructure during dynamic tensile extrusion, at $400 \mathrm{~m} \mathrm{~s}^{-1}$, of fully annealed OFHC copper with $15 \mu \mathrm{m}$ initial grain size, was investigated. The attention was focused on the analysis of the fragment that remained in the die, since its material points experience different deformation and temperature levels. Numerical simulation revealed that points along the axis of this fragment, represent different points along a common temperature compensated deformation history when plotted as $\log (Z)$ as a function of plastic strain. Therefore, post mortem microstructure information extracted at different locations is equivalent to in situ real-time measurement during the deformation process.

Microstructure evolution along the axis was investigated by means of extensive EBSD analysis, which showed a progressive elongation of the grains, and an accompanying development of a strong $<001>+<111>$ dual fibre texture. Meta-dynamic discontinuous dynamic recrystallization (mDRX) occurred at larger strains, and it was demonstrated that nucleation occurred during straining, while subsequent grain growth took place during post-deformation cooling in the die. According to strain energy minimization arguments, the recrystallization 
resulted in an increased <001> texture component. The on-set of recrystallization was well predicted by a phenomenological relationship, which relates the critical strain for DRX to the initial grain size and Zener-Hollomon parameter.

A texture dependence of the deformation substructure was observed from the EBSD data, and TEM investigations confirmed that $<001>$ and $<111>$ oriented grains develop different dislocation substructures. In the $<001>$ oriented regions elongated cells or microbands were observed, while the typical cell structure occurred in the $<111>$ oriented grains.

\section{ACKNOWLEDGEMENTS}

Authors MH, NM and MH wish to acknowledge the support from the Department of Applied Physics at Chalmers University of Technology for their part of the work. A grant from the Knut and Alice Wallenberg Foundation for acquiring the FEG-SEM instrument is gratefully acknowledged. Mr. Gabriel Testa is kindly acknowledged for performing the CPFEM calculations.

\section{REFERENCES}

[1] Murr LE, Niou C-S, Sanchez JC, Shih HK, Duplessis L, Pappu S, Zernow L. Journal of Materials Science 1995;30:2747.

[2] Chen W, Song B. Split Hopkinson (Kolsky) Bar: Design, Testing and Applications. New York: Springer, 2011.

[3] Gray III GT, Maudlin PJ, Hull LM, Zuo QK, Chen S-R. Journal of Failure Analysis and Prevention 2005;5:7.

[4] Maudlin PJ, Bingert JF, House JW, Chen SR. International Journal of Plasticity 1999;15:139.

[5] Rohr I, Nahme H, Thoma K. International Journal of Impact Engineering 2005;31:401.

[6] Iannitti G, Bonora N, Ruggiero A, Dichiaro S. Modeling ductile metals under large strain, pressure and high strain rate incorporating damage and microstructure evolution. 17th Biennial Conference of the American Physical Society Topical Group on Shock Compression of Condensed Matter - 2011 APS SCCM, vol. 1426. Chicago, 2012. p.1027.

[7] Anderson CEJ, Nicholls AE, Chocron IS, Ryckman RA. Taylor anvil impact. In: M. D. Furnish ME, T.P. Russell and T. White, editor. SHOCK COMPRESSION OF CONDENSED MATTER - 2005: Proceedings of the Conference of the American Physical Society Topical Group on Shock Compression of Condensed Matter, vol. 854 I, 2006. p.1367.

[8] Forde LC, Proud WG, Walley SM. Proceedings of the Royal Society of London. Series A, Mathematical and Physical 2009;465:769.

[9] Gray III GT, Cerreta E, Yablinsky CA, Addessio LB, Henrie BL, Sencer BH, Buckett M, Maudlin PJ, Maloy SA, Trujillo CP, Lopez MF. Influence of Shock Prestraining and Grain Size on the Dynamic-Tensile-Extrusion Response of Copper: Experiments and Simulation. SHOCK 
COMPRESSION OF CONDENSED MATTER - 2005: Proceedings of the Conference of the American Physical Society Topical Group on Shock Compression of Condensed Matter, vol. 845 I, 2006. p.725.

[10] Gray III GT, Cerreta EK, Yablinsky CA, Addessio LB, Henrie BL, Sencer BH, Burkett M, Maudlin PJ, Maloy SA, Trujillo CP, Lopez MF. 2006;845:725.

[11] Cao F, Cerreta EK, Trujillo CP, Gray III GT. Acta Materialia 2008;56:5804.

[12] Escobedo JP, Cerreta EK, Trujillo CP, Martinez DT, Lebensohn RA, Webster VA, Gray III GT. Acta Materialia 2012;60:4379.

[13] Furmanski J, Trujillo CP, Martinez DT, T. GIG, Brown EN. Polymer Testing 2012;31:1031.

[14] Park K-TP, Park L, Kim HJ, Kim SB, Lee CS. Material Science and Engineering A 2013;569:61.

[15] Flater PJ. Personal communication. 2009.

[16] Carlucci P, Mougeotte C, Huidi J. Validation of ABAQUS Explicit - CEL for classes of problems of interest to the U.S. Army. SIMULIA Customer Conference. Providence, Rod Island (USA), 2010. p.1.

[17] Iannitti G, Bonora N, Ruggiero A, Dichiaro S. Modeling ductile metals under large strain, pressure and high strain rate incorporating damage and microstructure evolution 17 th Biennial Conference of the American Physical Society Topical Group on Shock Compression of Condensed Matter, 2011 APS SCCM, vol. 1426. Chicago, IL, 2012. p.1027.

[18] Armstrong RW, Walley SM. International Materials Reviews 2004;53:105.

[19] Bonora N, Ruggiero A, Flater PJ, House JW, Deangelis RJ. On the role of material postnecking stress-strain curve in the simulation of dynamic impact. SHOCK COMPRESSION OF CONDENSED MATTER - 2005: Proceedings of the Conference of the American Physical Society Topical Group on Shock Compression of Condensed Matter, vol. 845 I. Baltimore, MD, 2006. p.701.

[20] Follansbee PS, Regazzoni G, Kocks UF. The transition to drag-controlled deformation in copper at high strain rates. In: Harding J, editor. Third International Conference on Mechanical Properties of Materials at High Strain Rates, vol. 70, 1984. p.71.

[21] Follansbee PS, Weertman J. Mechanics of Materials 1982;1:345.

[22] Regazzoni G, Kocks UF, Follansbee PS. Acta Materialia 1987;46:565.

[23] Tiez TE, Dorn JE. Journal of Metals 1956;206:156.

[24] Feltham P, Meakin JD. Acta Metallurgica 1959;7:614.

[25] Barrett CR, Sherby OD. Transaction of Metallurgical Society AIME 1964;230:1322.

[26] Freed AD, Raj SV, Walker KP. Journal of Engineering Materials and Technology Transaction of ASME 1992;114:46.

[27] Furushiro N, Okada Y, Hori S. Journal of the Society of Materials Science Japan 1980;29:776.

[28] Blaz L, Sakai T, Jonas JJ. Metal Science 1983;17:609.

[29] Bonora N, Ruggiero A, Testa G, Hörnqvist M, Mortazavi Seyedeh N, Halvarsson M. International Journal of Plasticity 2014;Submitted. 
[30] Gao W, Belyakov A, Mura H, Sakai T. Material Science and Engineering A 1999:233.

[31] Birosca S, Di Gioacchino F, Stekovic S, Hardy M. Acta Materialia 2014;74:110.

[32] Raabe D, Zhao Z, Mao W. Acta Materialia 2002;50:4379.

[33] Cronje S, Kroon RE, Roos WD, Neethling JH. Bullettin of Materials Science 2013;36:157.

[34] Hong CS, Tao NR, Lu K, Huang X. Scripta Materialia 2009;61:289.

[35] Naaman H, Talreja R, Jensen DJ, Hansen N. Textures and Microstructure 1987;7:149.

[36] Ahlborn H. Z. Metallkunde 1965;56:411.

[37] Waryoba DR, Kalu PN. Textural inhomogeneities in drawn and annealed OFHC copper wire TMS Annual Meeting, 2005. p.247.

[38] Waryoba DR, Kalu PN. Material Science Forum 2005:877.

[39] Kalu PN, Waryoba DR. Material Science Forum 2007;550:509.

[40] Leffers T. Scripta Metallurgica 1968;2:447.

[41] Leffers T, Pedersen OB. Material Science Forum 2002;408-412(I):607.

[42] Bhattacharyya A, Rittel D, Ravichandran G. Scripta Materialia 2005;52:657.

[43] Park LJ, Kim HW, Lee CS, Park K-T. Materials Transactions 2010;51:2049.

[44] https://icme.hpc.msstate.edu/mediawiki/index.php/Code:_ABAQUS_CPFEM.

[45] Bachmann F, Hielscher R, Schaeben H. Solid State Phenomena 2010;160:63.

[46] Marin EB, Dawson PR. Computer methods in applied mechanics and engineering 1998;165:23.

[47] Lebensohn RA, Tome C. Acta Metallurgica Materialia 1993;41:2611.

[48] Molinari A, Canova GR, Ahzi S. Acta Metallurgica 1987;35:2983.

[49] Li S, Kalidindi SR, Beyerlein IJ. Materials Science and Engineering: A 2005;410-411:207.

[50] Lee DN. Scripta Metallurgica et Materialia 1995;32:1689.

[51] Shin H-J, Jeong H-T, Lee DN. Materials Science and Engineering: A 2000;279:244.

[52] Humphreys FJ, Hatherly M. Recrystallization and Related Annealing Phenomena, Second Edition. Kidlington, Oxford, UK: Elsevire Ltd., 2004.

[53] Cram DG, Fang XY, Zurob HS, Bréchet YJM, Hutchinson CR. Acta Materialia 2012;60:6390.

[54] Cram DG, Zurob HS, Brechet YJM, Hutchinson CR. Acta Materialia 2009;57:5218.

[55] Turnbull D. Transactions of AIME 1951;191:661.

[56] Bailey JE, Hirsch PB. Proceedings of the Royal Society of London. Series A, Mathematical and Physical Sciences 1962;267:11.

[57] Gubicza J, Balogh L, Hellmig RJ, Estrin Y, Ungár T. Materials Science and Engineering: A 2005;400-401:334.

[58] Murata Y, Nakaya I, Morinaga M. Materials Transactions 2008;49:20.

[59] Petkovic RA, Luton MJ, Jonas JJ. Acta Metallurgica 1979;27:1633.

[60] Nadal M-H, Le Poac P. Journal of Applied Physics 2003;93:2472. 


\section{FIGURE}
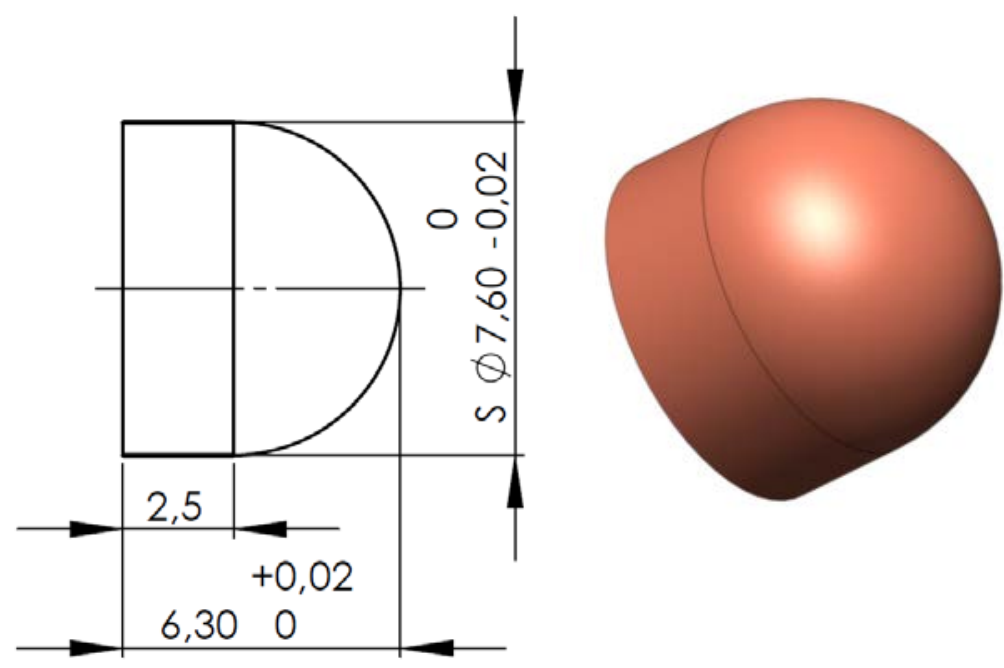

Figure 1 -DTE bullet: dimensions in $\mathrm{mm}$ and 3D sketch.

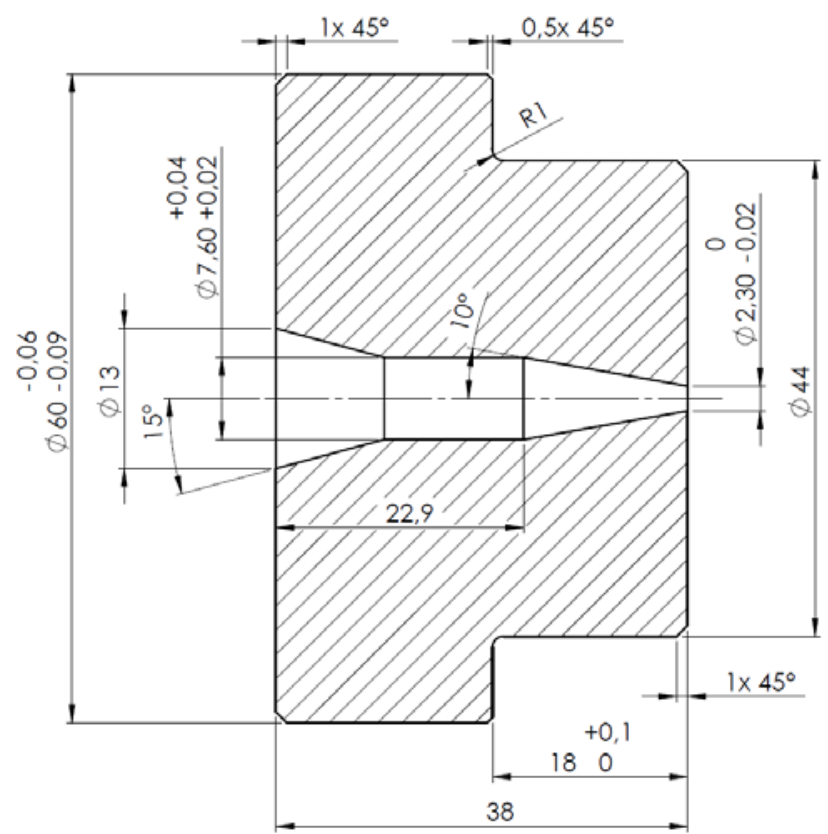

Figure 2 -DTE die dimensions in mm. 


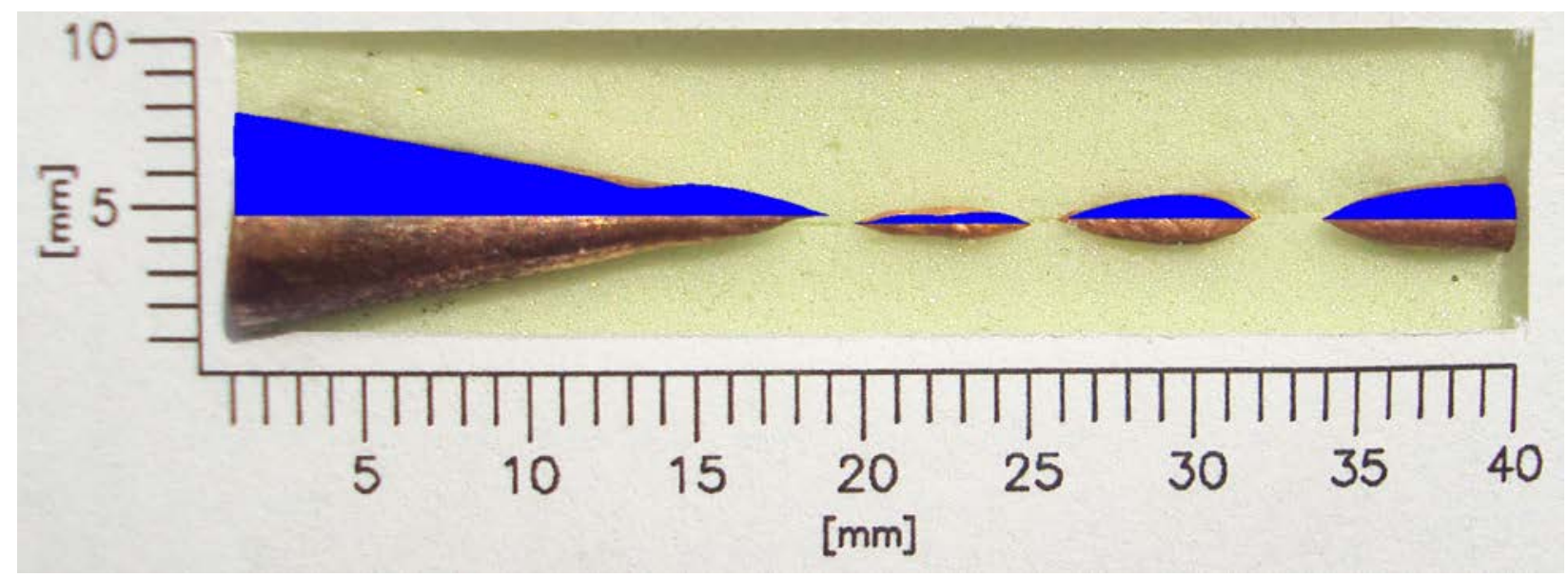

a) mJ-A model

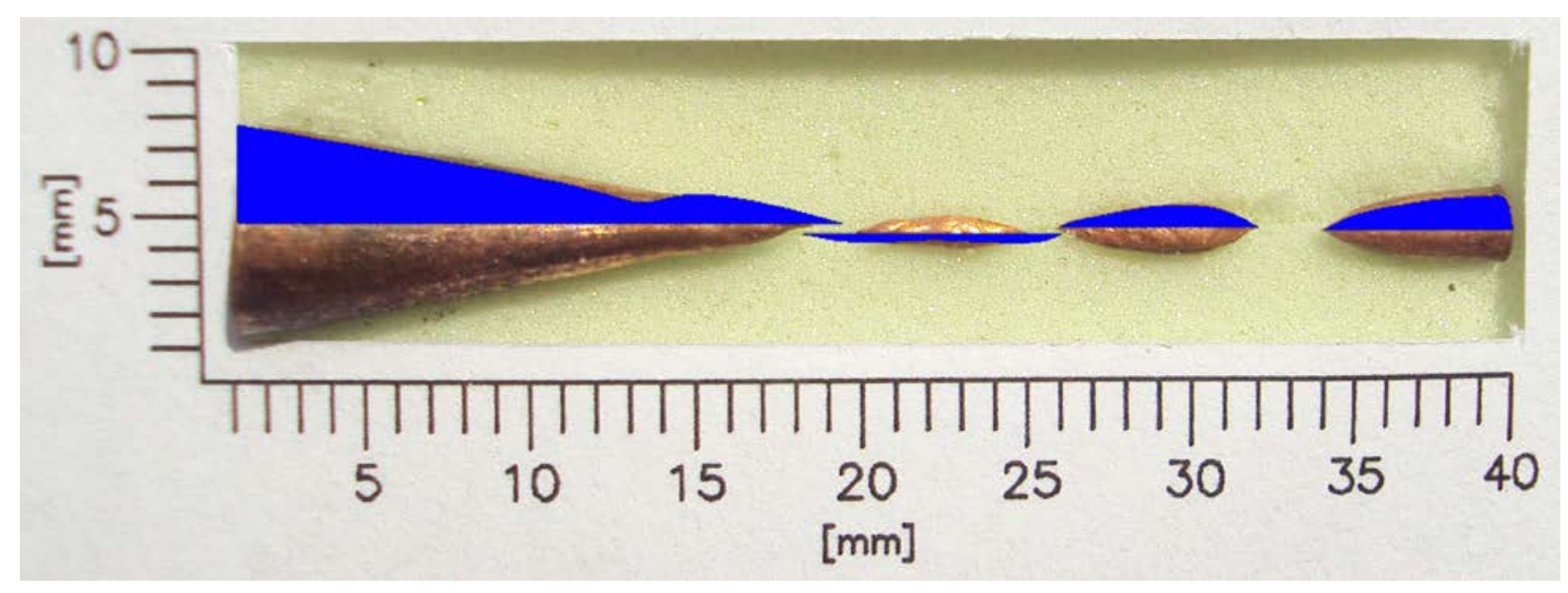

mZ-A model

Figure 3 -Comparison of the predicted fragments shape using mJ-C and mZ-A strength models.
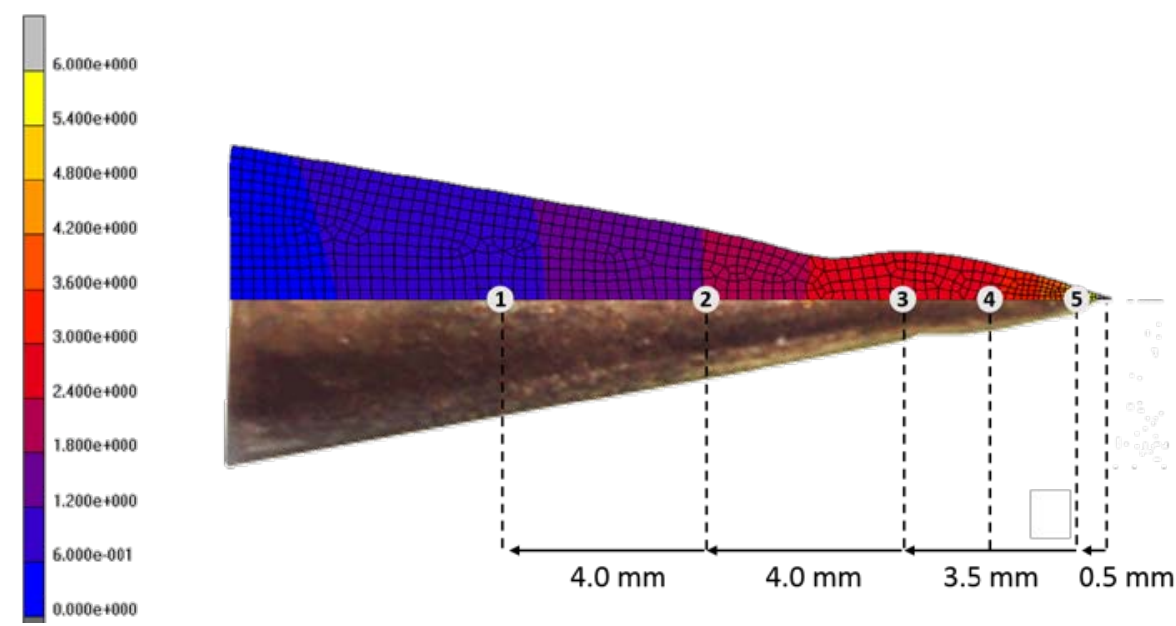

Figure 4 -Location of selected sample points for FEM results and EBDS. Contour plot represent plastic strain contours with mJ-C model. 

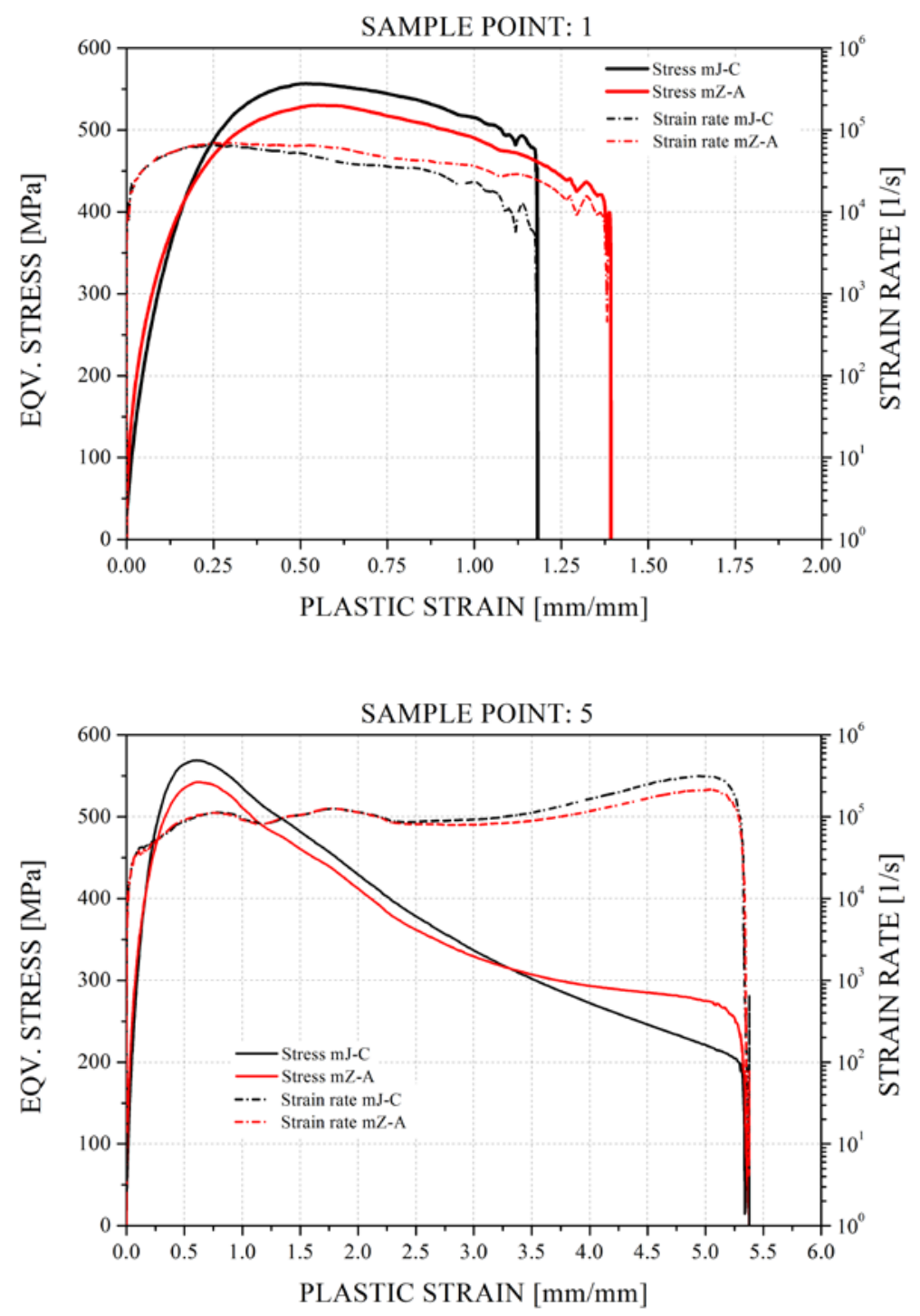

Figure 5 -Comparison of calculated von Mises stress and strain rate vs plastic strain for mJ$\mathrm{C}$ and $\mathrm{mZ}-\mathrm{A}$ strength models for sample point 1 and 5 . 


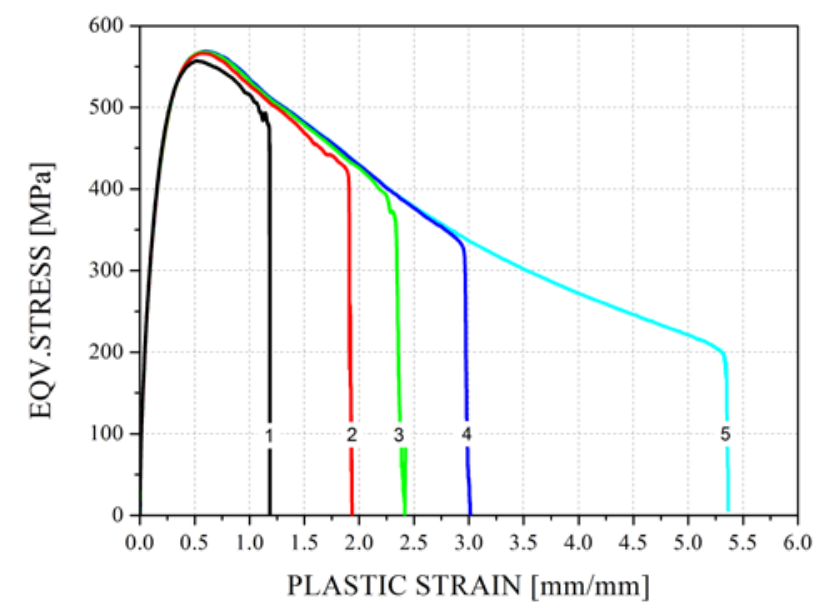

Figure 8

a)

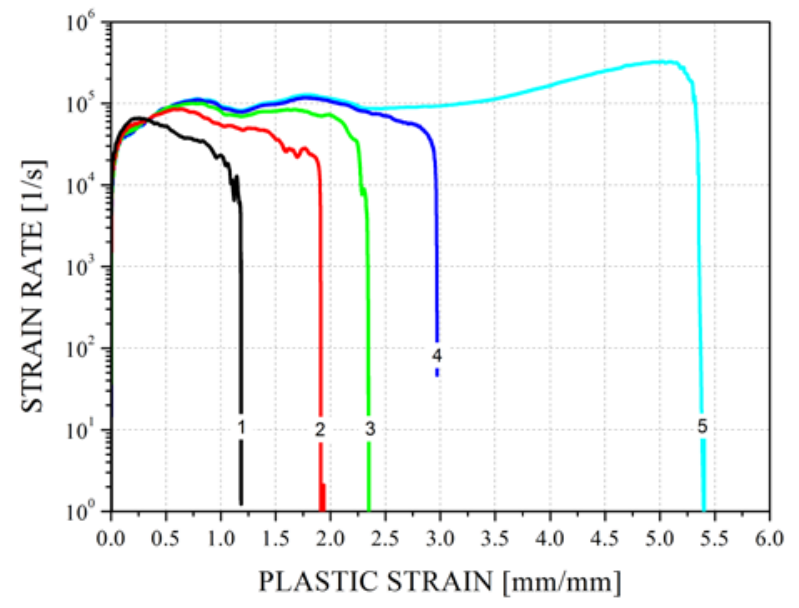

b)

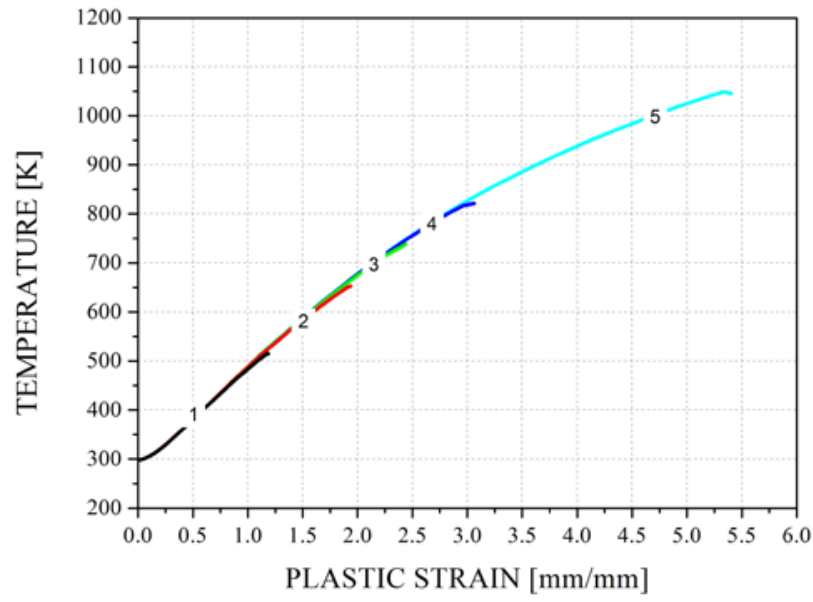

c)

Figure 6 -Summary of a) stress, b) strain rate and c) temperature vs plastic strain for all selected locations along the fragment symmetry axis (mJ-C model). 


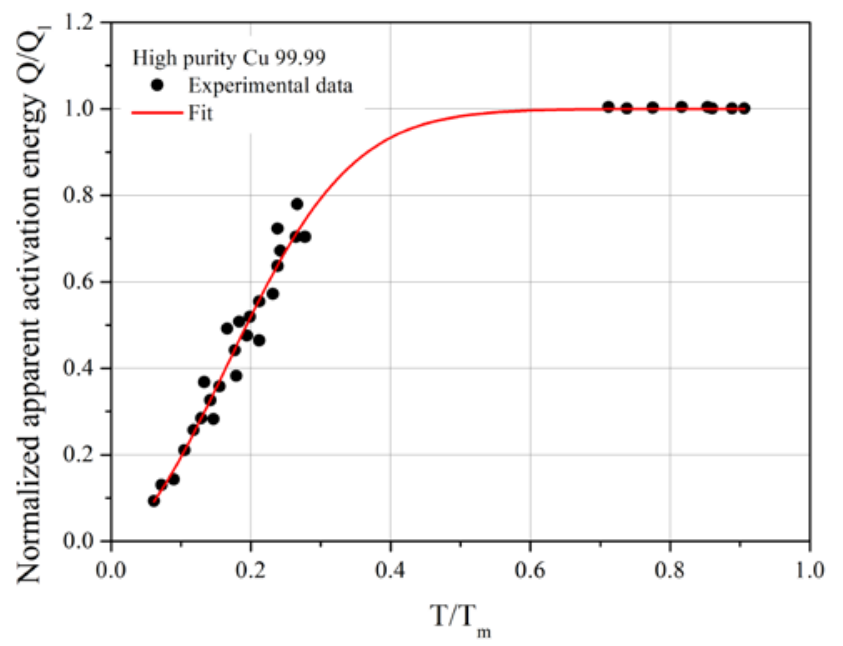

Figure 7 - Variation of apparent activation energy with temperature. Here $Q_{l}=210 \mathrm{~kJ} \mathrm{~mol}^{-1}$. Experimental data are taken from [23-25].

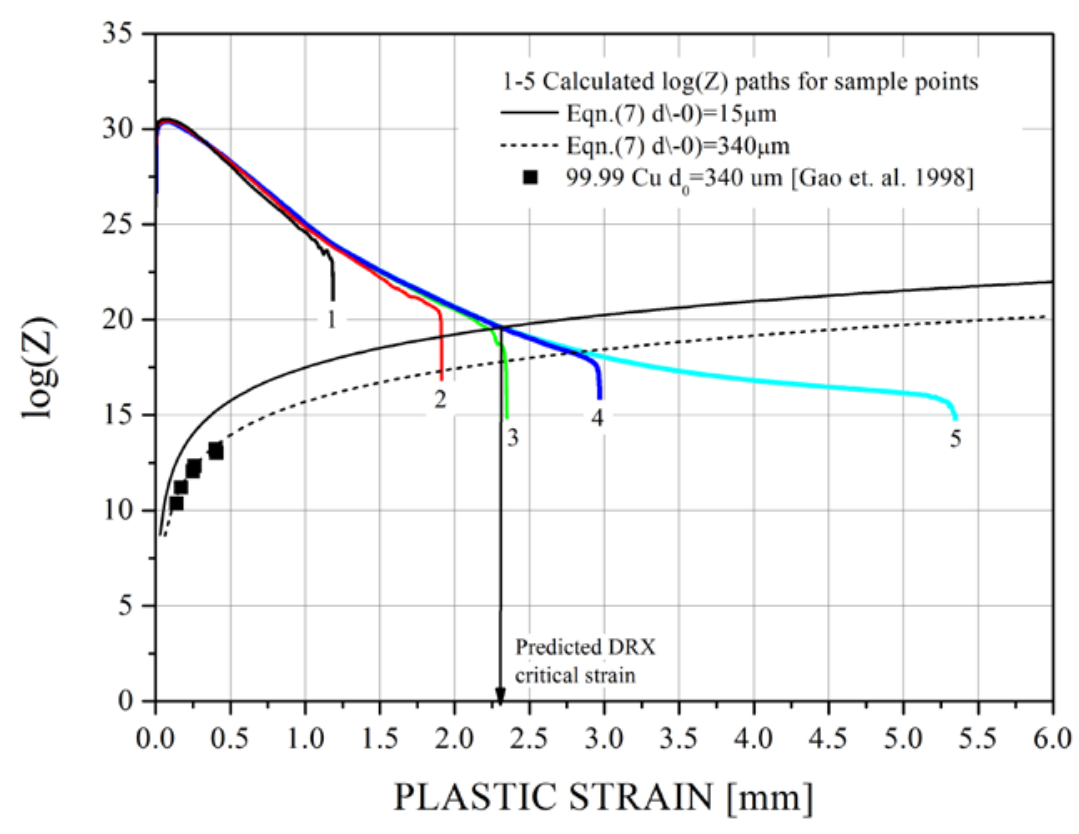

Figure 8 - Calculated $\log (Z)$ paths for sample points 1 to 5 as a function of plastic strain. DRX critical strain for $15 \mu \mathrm{m}$ initial grain size is determined as the intersection of Eqn. (7) with $\log (Z)$ path. 


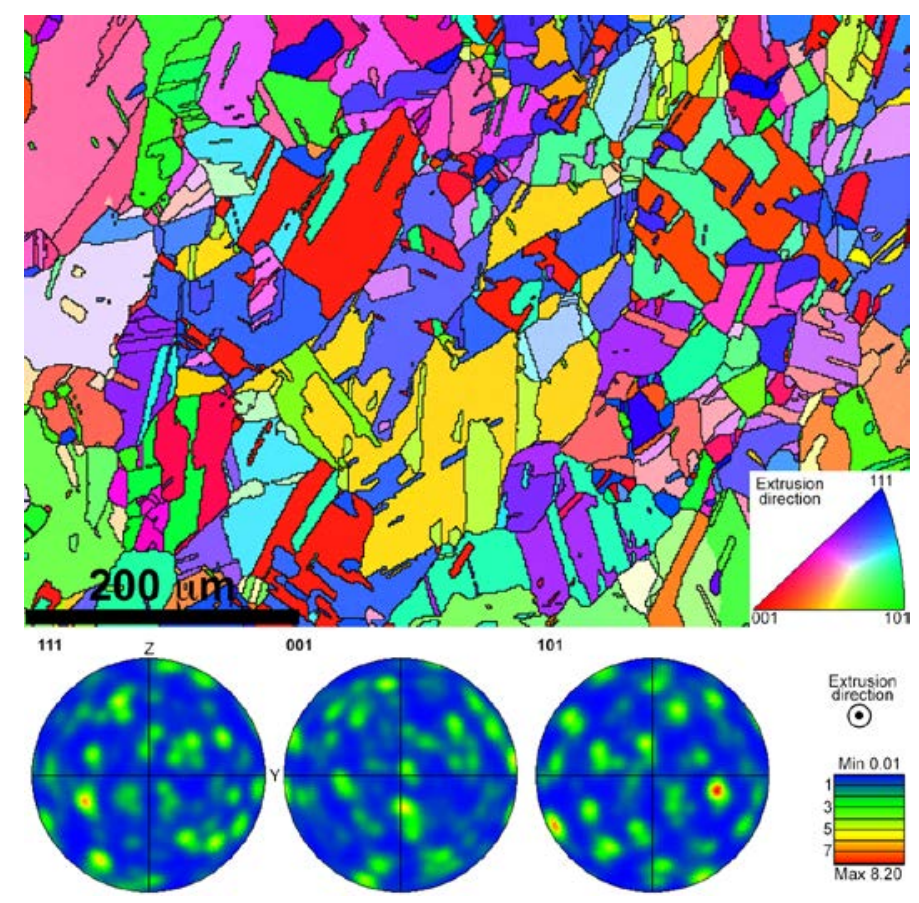

Figure 9 - Inverse pole figure map and pole figures for the annealed material. The inverse pole figure map shows orientations aligned with the extrusion direction, which is horizontal in the figure. The pole figures show a random initial texture.
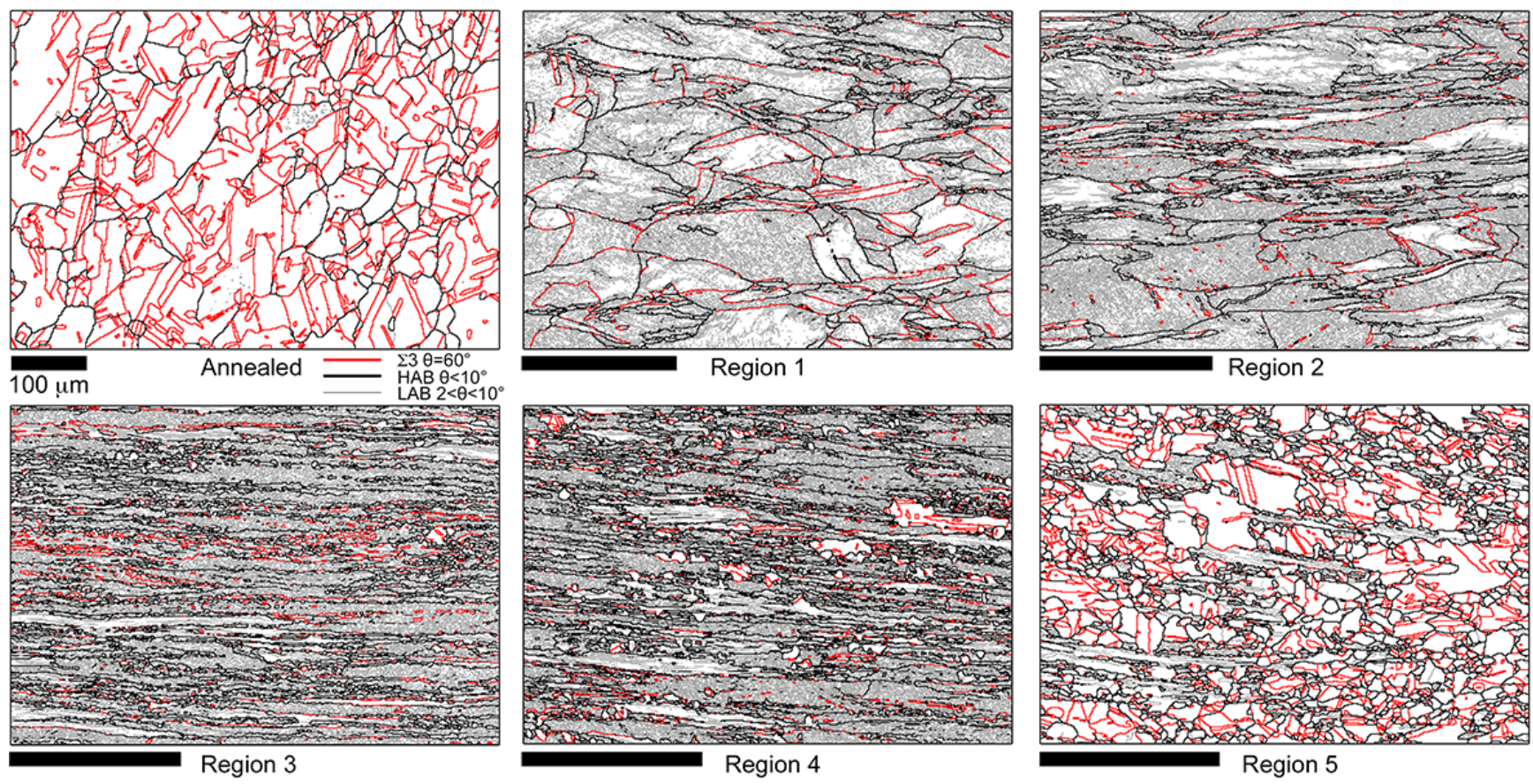

Figure 10 - Grain structure from the EBSD investigations of the annealed sample and different regions in the DTE specimen. 

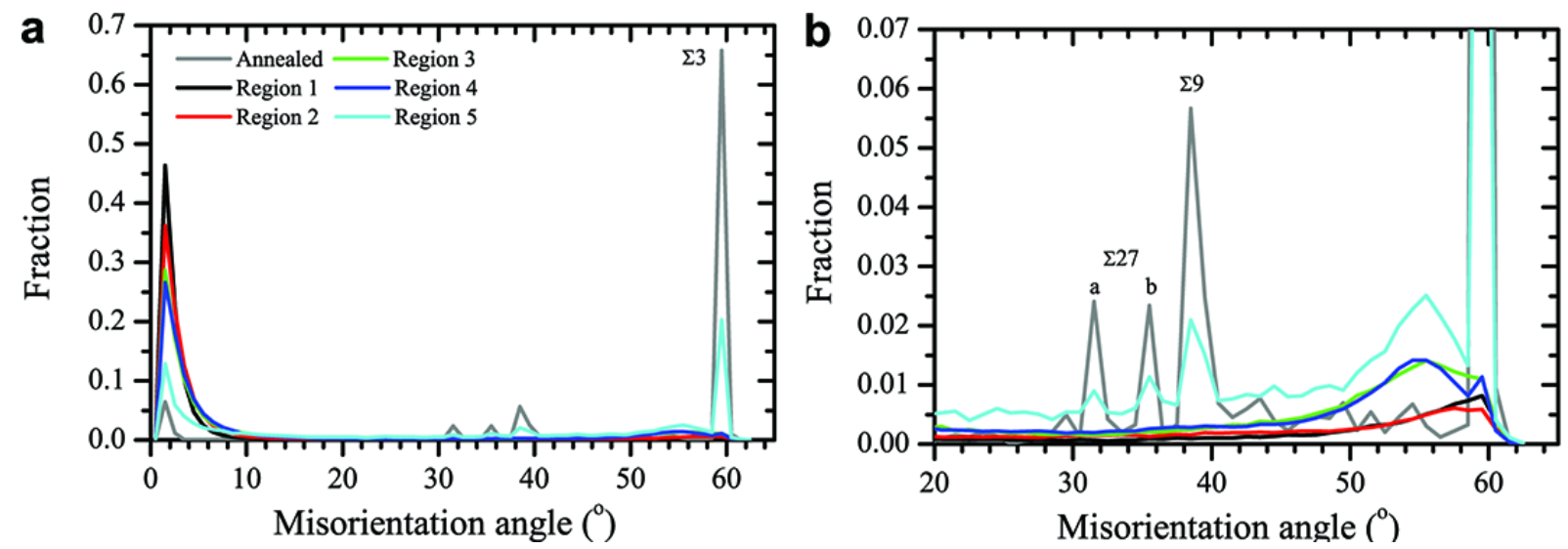

Figure 11 - (a) Misorientation angle distribution for the different regions. (b) Close-up showing the presence of other CSL boundaries in the annealed state, and in the final region (plastic strain of 5.4).

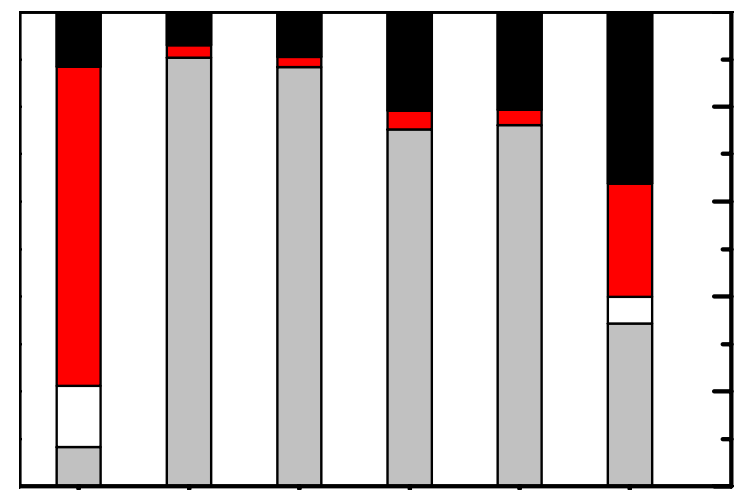

Figure 12 - Fraction of grain boundary types in the different regions. 


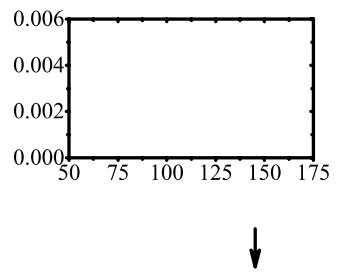

m)
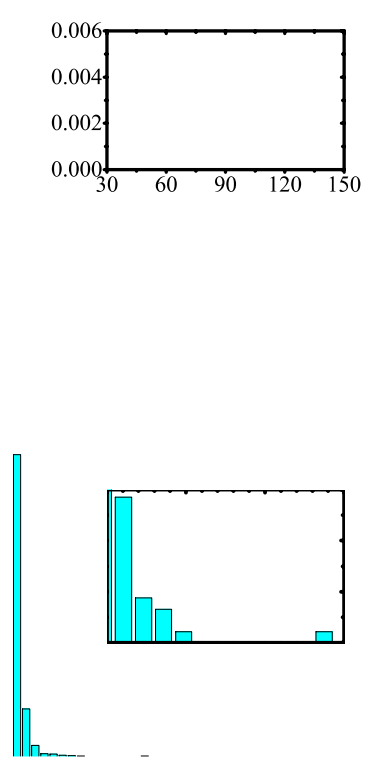

Figure 13 - Histograms of the grain size from the different sample points (here indicated as "regions") (including twin boundaries, and corrections for edge intersection). The insets show magnifications of the distribution tail, and the arrows indicate the maximum measured grain size.

a

$\mathrm{n}$ size $(\mu \mathrm{m})$

Figure 14 - Same as in Figure 13, but based on area fractions instead of number fractions. 


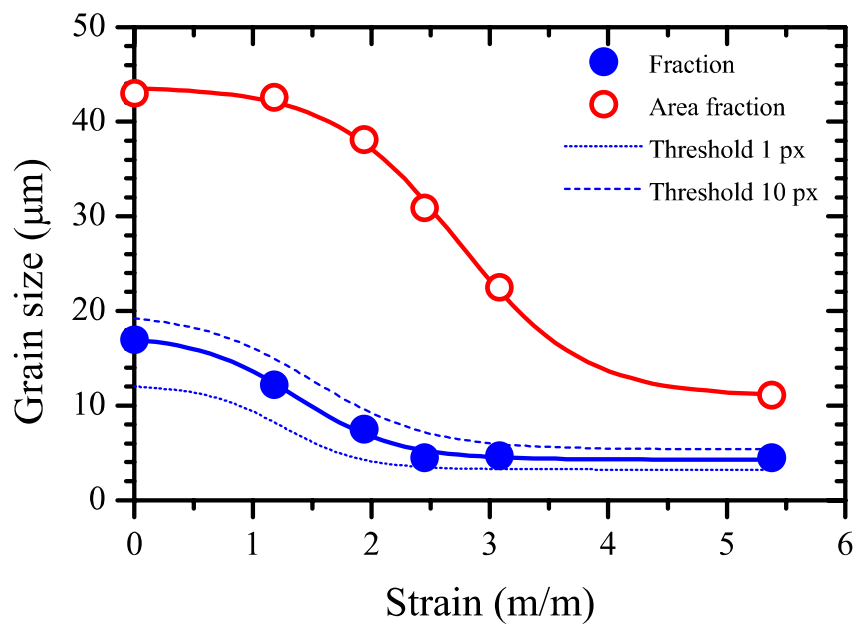

Figure 15 - Evolution of the average grain size, based on both number fraction and area fraction, with strain. Also included are the limits corresponding to a threshold for inclusion of 1 pixel and 10 pixels. The average curves are based on a minimum of 5 pixels in a grain.
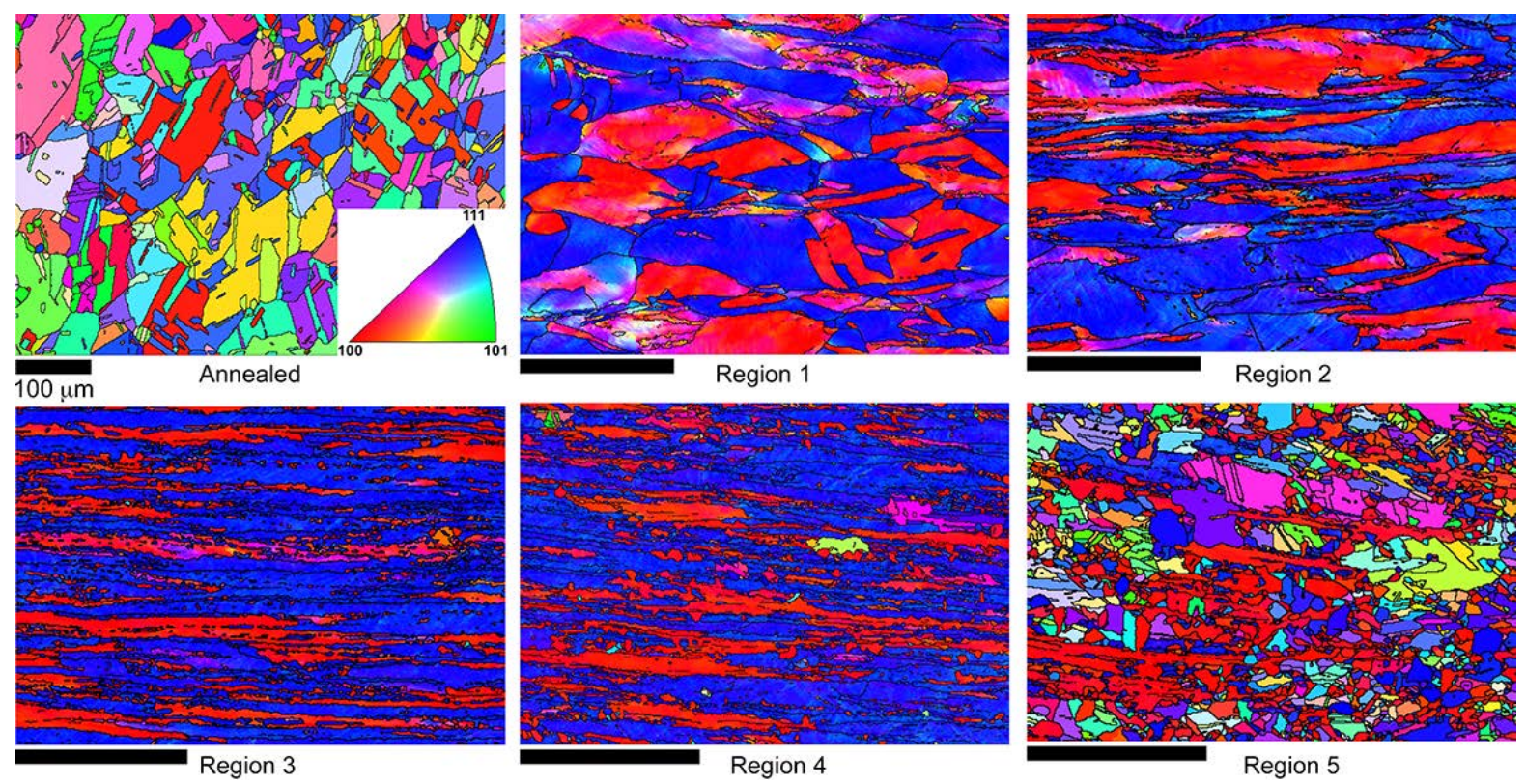

Figure 16 - IPF maps of the annealed sample and different regions in the DTE specimen. All scale bars are $100 \mu \mathrm{m}$. 

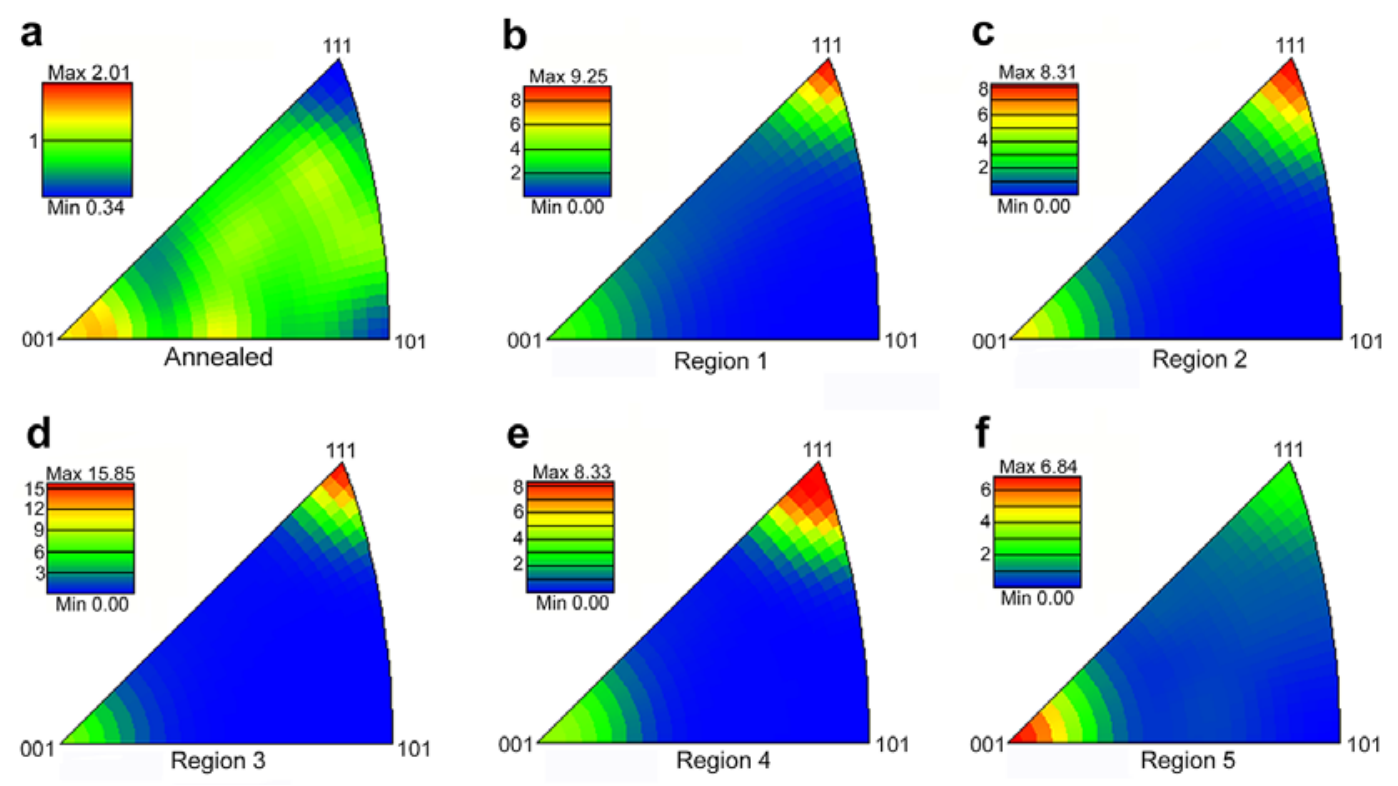

Figure 17 - IPFs from the EBSD maps of the different regions showing (a) the initially random texture; (b-e) the development of a strong dual $<001>+<111>$ fibre texture, dominated by the $<111>$ fibre; and (f) changes in the relative fibre strengths due to recrystallization of predominantly $<111>$ oriented regions into $<001>$ orientations.

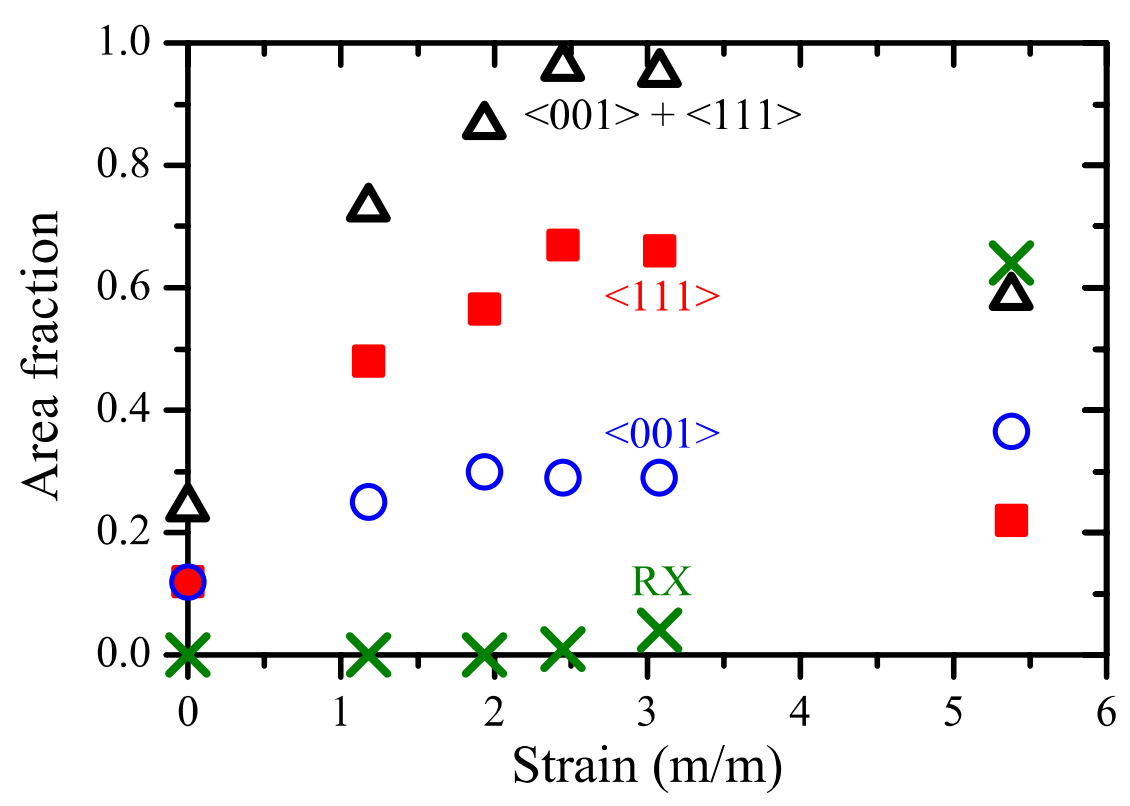

Figure 18 - Evolution of the fraction of different texture components and recrystallized grains $(\mathrm{RX})$ with strain. 


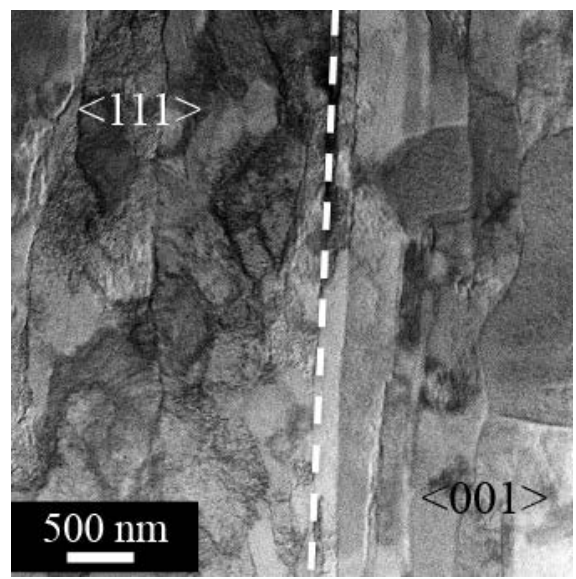

Figure 19 - TEM micrographs of the dislocation structure in adjacent $<111>$ and $<001>$ grains. The grain boundary is indicated by the dashed line.
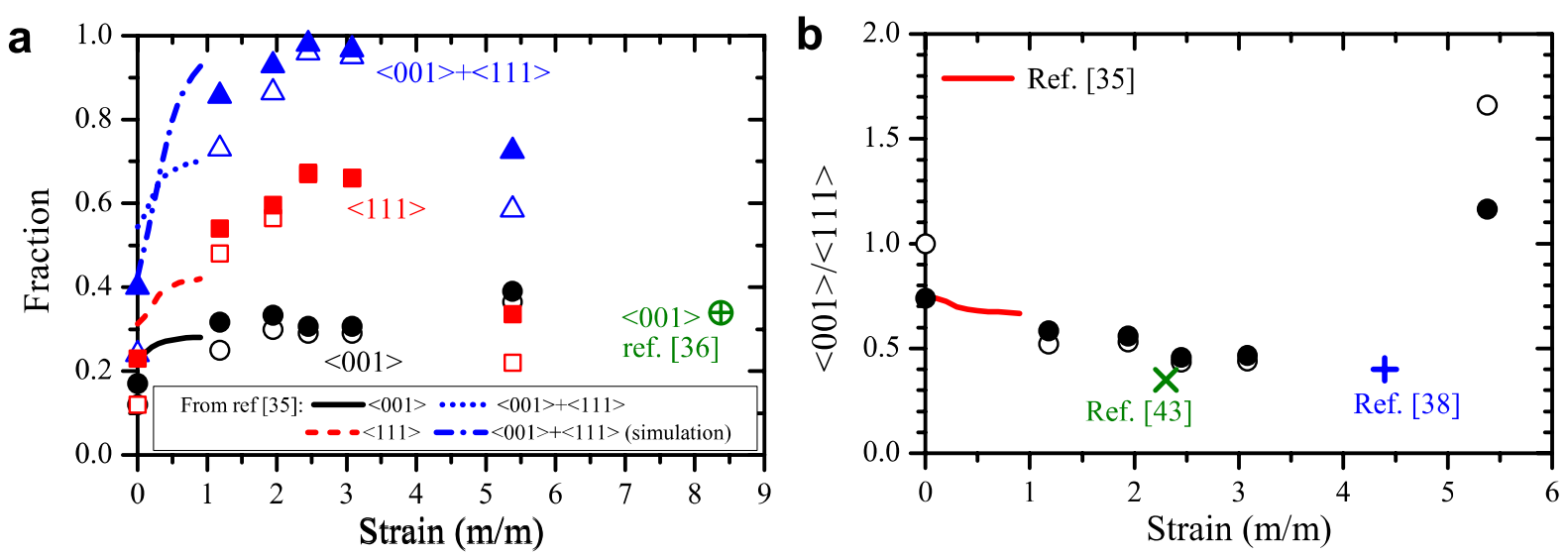

Figure 20 - Evolution of texture strength with strain. (a) Fractions of $<001>,<111>$ and $<001>+<111>$ fibres. (b) Ratio $<001>/<111>$. Open and closed symbols denote integration over $15^{\circ}$ and $20^{\circ}$, respectively.
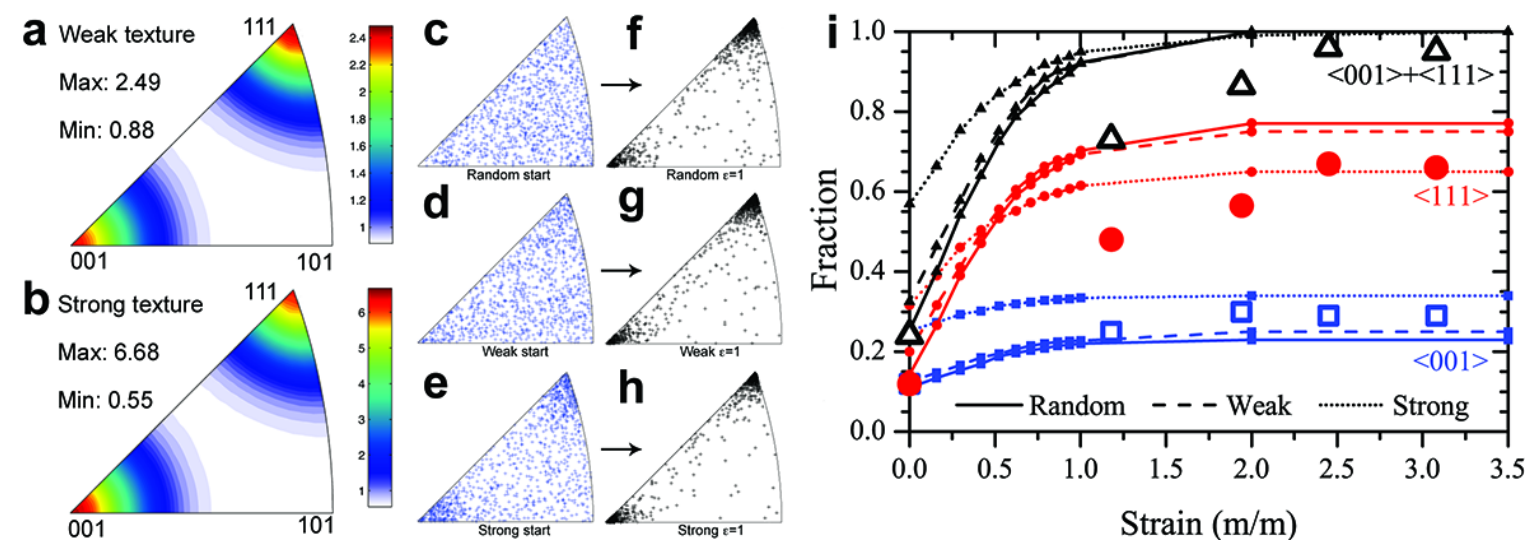

Figure 21 - Synthetic IPF figures for (a) the weak, and (b) the strong texture. (c), (d) and (e) shows the generated sets of orientations generated for the random, weak and strong starting textures, respectively. (f-h) shows the resulting orientations after a strain of 1 . (i) shows the development of the volume fractions of the $<001>,<111>$ and $<001>+<111>$ fibres with strain, compared to the experimental data. 


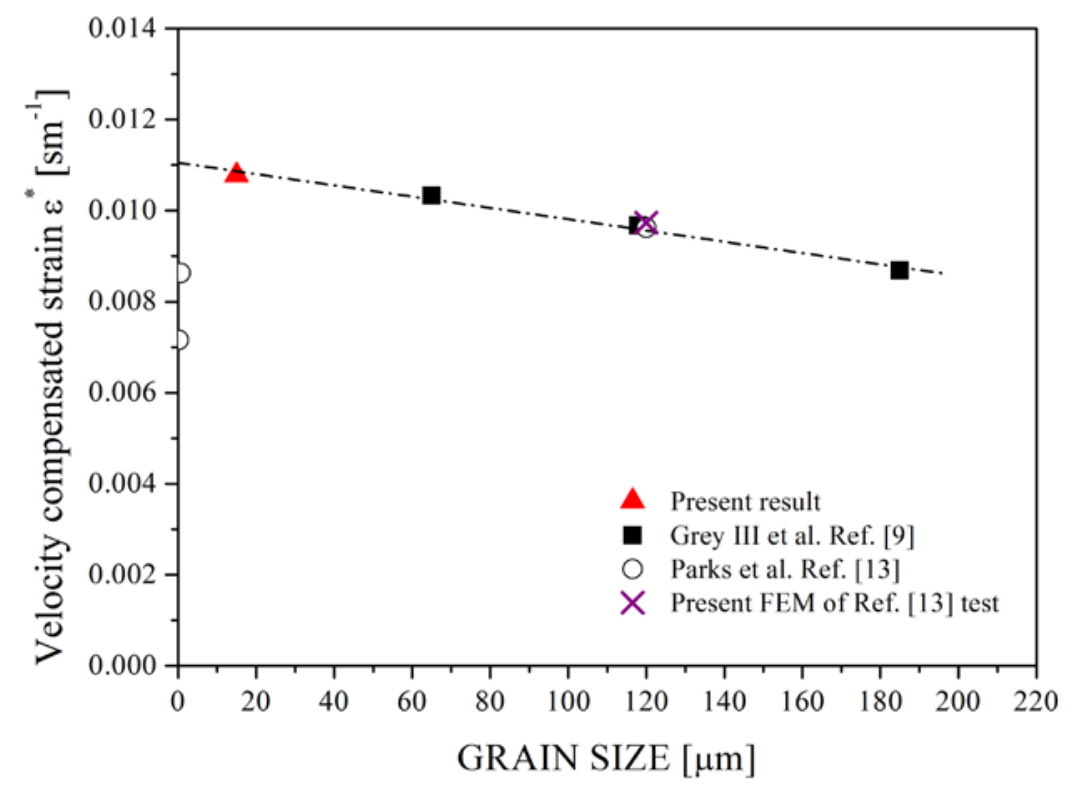

Figure 22 - Velocity compensated engineering strain as a function of initial grain size.

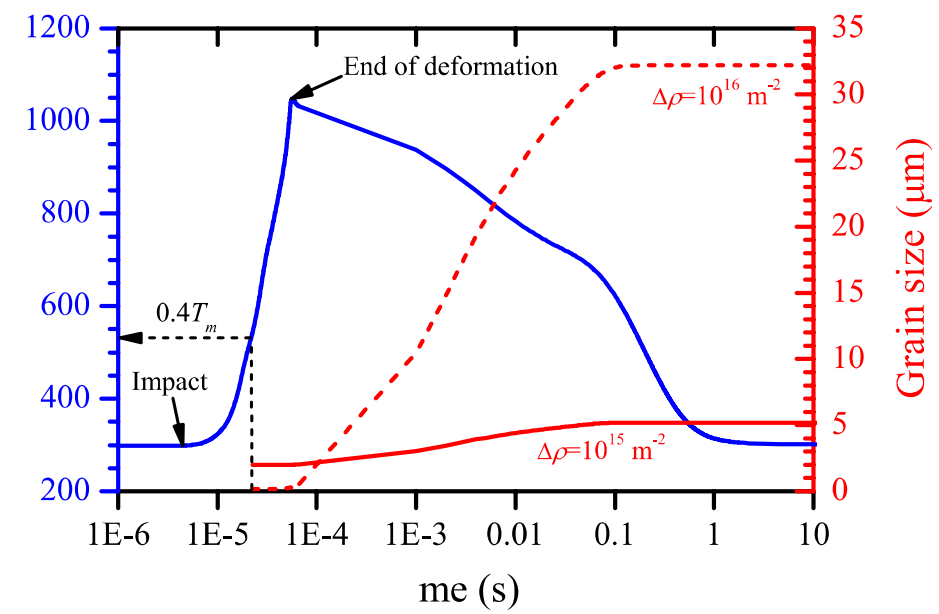

Figure 23 - Temperature from numerical simulation region 5 throughout of the entire test, including cooling inside the die, and evolution of the recrystallized grain size. 
Table 1.Parameters in the modified Johnsson-Cook (mJ-C) and modified Zerilli-Armstrong (mZA) models.

\begin{tabular}{|c|c|c|}
\hline mJ-C model & Value & Units \\
\hline$\sigma_{y}^{0}$ & 21.8 & $\mathrm{MPa}$ \\
\hline$B_{1}$ & 36.5 & $\mathrm{MPa}$ \\
\hline$B_{2}$ & 357.5 & $\mathrm{MPa}$ \\
\hline$t_{1}$ & 0.0205 & - \\
\hline$t_{2}$ & 0.175 & - \\
\hline $\mathrm{C}$ & 0.046 & - \\
\hline$\dot{\varepsilon}_{0}$ & 1.0 & $\mathrm{~s}^{-1}$ \\
\hline$T_{m}$ & 1356 & $\mathrm{~K}$ \\
\hline$m$ & 1.09 & - \\
\hline
\end{tabular}

\begin{tabular}{|c|c|c|}
\hline mZ-A model & Value & Units \\
\hline$C_{0}$ & 21.8 & $\mathrm{MPa}$ \\
\hline$C_{2}$ & 1750 & $\mathrm{MPa}$ \\
\hline$C_{3}$ & 0.0028 & $\mathrm{~K}^{-1}$ \\
\hline$C_{4}$ & $1.15 \mathrm{E}-04$ & $\mathrm{~K}^{-1}$ \\
\hline$\varepsilon_{r}$ & 0.35 & - \\
\hline
\end{tabular}

Table 2. Parameters in Eq. (RX4).

\begin{tabular}{|c|c|c|c|}
\hline Parameter & Value & Units & Reference \\
\hline$\beta$ & 0.2 & - & [53] \\
\hline$\delta$ & $1 \cdot 10^{-9}$ & $\mathrm{~m}$ & {$[53]$} \\
\hline$D_{0, g b}$ & $2.35 \cdot 10^{-5}$ & $\mathrm{~m}^{2} \mathrm{~s}^{-1}$ & {$[53]$} \\
\hline$Q_{g b}$ & $107.2 \cdot 10^{3}$ & $\mathrm{~J} \mathrm{~mol}^{-1}$ & {$[53]$} \\
\hline$\Omega_{m}$ & $7.11 \cdot 10^{-6}$ & $\mathrm{~m}^{3} / \mathrm{mol}$ & {$[60]$} \\
\hline$\mu$ & $50.7 \cdot 10^{9} \cdot\left(1-0.45 \cdot T / T_{\mathrm{m}}\right)$ & $\mathrm{Pa}$ & {$[54]$} \\
\hline$\gamma$ & 0.625 & $\mathrm{~J} \mathrm{~m}^{-2}$ & \\
\hline
\end{tabular}

